\title{
Morphological pseudotime ordering and fate mapping reveals diversification of cerebellar inhibitory interneurons
}

\section{Wendy Xueyi Wang ${ }^{1,2}$ and Julie L. Lefebvre',2*}

${ }^{1}$ Department of Molecular Genetics, University of Toronto, Toronto, ON, Canada; ${ }^{2}$ Program for Neuroscience and Mental Health, Hospital for Sick Children Research Institute, Toronto, ON, Canada

1 Abstract Understanding how diverse neurons are assembled into circuits requires a

2 framework for describing cell types and their developmental trajectories. Here, we combined

3 genetic fate mapping and pseudo-temporal profiling to resolve the diversification of cerebellar

4 inhibitory interneurons based on morphology. The molecular layer interneurons (MLIs) derive

5 from a common progenitor but comprise a diverse population of dendritic-, somatic-, and

6 axon initial segment-targeting interneurons. MLls are classically divided into two types.

7 However, their morphological heterogeneity suggests an alternate model of one continuously

8 varying population. Through clustering and trajectory inference of $811 \mathrm{MLI}$ reconstructions at

9 maturity and during development, we show that MLIs divide into two discrete classes but also

10 present significant within-class heterogeneity. Pseudotime trajectory mapping uncovered the

11 emergence of distinct phenotypes during migration and axonogenesis, well before neurons

12 reach their final positions. Our study illustrates the utility of quantitative single-cell methods

13 to morphology for defining the diversification of neuronal subtypes.

*For correspondence:

julie.lefebvre@sickkids.ca (JLL)

Competing interests: The authors declare that no competing interests exist.

Acknowledgements: see page 27

Contributions: see page 28

\section{Introduction}

15 Neuronal diversification is essential for the assembly and function of 16 complex nervous systems (Zeng and Sanes 2017; Cembrowski and 17 Spruston 2019; Geirsdottir et al. 2019). The core feature is the divergence 18 of neuronal populations from shared lineages to subtypes with distinctive 19 features, spatial arrangements, and connectivity patterns (Lefebvre et 20 al. 2015). Subtype diversity arises from the interplay of intrinsic genetic

21 programs and extrinsic cues but capturing the underlying mechanisms

22 remains challenging. By profiling neurons across development with

23 single-cell transcriptomics, one can trace cell fate trajectories based on 24 gene expression signatures (Mayer et al. 2018; Mi et al. 2018; Clark et 25 al. 2019; Tiklová et al. 2019; Trapnell et al. 2014). These studies provide 26 insights into the dynamics and complexity of differentiating neural tissues, 27 including lineage relationships, intermediate progenitors, timelines 28 of maturation and the emergence of subtype identities (Trapnell et al. 29 2014; Saelens et al. 2019). However, charting neuronal diversification 30 requires additional modalities, such as morphology, to pinpoint emergent

31 structural features and to identify relevant developmental factors. 
Although morphology as a modality for cell typing is low throughput, it provides intuitive information about the identity, ontogeny and connectivity of neurons (Gouwens et al. 2019; Scala et al. 2020; Wang et al. 2019; Winnubst et al. 2019). New technologies are advancing the resolution and throughput for mapping cell types based on quantitative morphology (Costa et al. 2016; Winnubst et al. 2019; Economo et al. 2016; Sümbül et al. 2014; Markram et al. 2015; Wang et al. 2019). Correspondingly, large-scale morphological surveys are revealing constituent cell types and providing insights into connectivity patterns and circuit organization (Bae et al. 2018; Helmstaedter et al. 2013; Gouwens et al. 2019; Frechter et al. 2019; Jiang et al. 2015). On the other hand, studies focused on single or related cell types can provide detailed views of local anatomical variation and subtype diversification. This approach requires sparse labeling of genetically or lineage-related cell types, which is facilitated by transgenic drivers and viral vectors (Harris et al. 2014; He et al. 2016). Paired with advancements in statistical and computational methods, neuronal subtypes can be effectively parsed using single neuron anatomy, as illustrated for sensory afferents in the skin (Wu et al. 2012), olfactory bulb neurons (Tavakoli et al. 2018), and pyramidal and GABAergic interneurons in the cortex (Wang et al. 2019; Kanari et al. 2019; Gouwens et al. 2019). Drawing fine divisions between subtypes can be challenging however, as heterogeneity and continuous variation within cell types are commonly observed (Cembrowski and Menon 2018; Gouwens et al. 2019; Harris et al. 2018; Muñoz-Manchado et al. 2018; Scala et al. 2020). Therefore, a major question driving studies of neural diversity is whether all cells sort into discrete subtypes, given sufficient sampling and granularity, or if continuous and local variation is a biological feature essential for neural processing. Large-scale, unbiased morphological analyses have yet to be exploited to study how variation arises during development.

Here, we test a pipeline for mapping the diversification of cerebellar GABAergic interneurons using quantitative morphology. Compared to the complexity of forebrain interneurons (Huang and Paul 2019), the cerebellar molecular layer interneurons (MLIs) derive from a single lineage and form simpler, compact morphologies (Palay and Chan-Palay 1974; Sotelo 2015). Nonetheless, MLIs form an anatomically and functionally diverse population that provides the full complement of dendritic-, somatic- and axon initial segment-targeting inhibition onto principal Purkinje cells. Classically, MLIs are divided into two types—basket cells and stellate cells (Palay and Chan-Palay 1974). Basket cells (BCs) are born earlier, populate the lower third of the molecular layer and form a series of perisomatic basket terminals that envelope the Purkinje cell soma. Some BC terminals further specialize into pinceaux formations that align the Purkinje cell axon initial segment (AIS; Buttermore et al. 2012; Sotelo 2015). By contrast, later-born stellate cells (SCs) settle in the upper molecular layer where their axons innervate Purkinje cell dendrites. However, this classical division has long been debated due to continuous morphological variation observed among MLIs (Sotelo 2015; Rakic 1972; Sultan and Bower 1998). As suggested by fate mapping and heterochronic transplantation studies, the laminar gradient of BC-to-SC phenotypes is related to birthdate and the inside-out settling of precursors within the cerebellar cortex (Altman and Bayer 1978; Cameron 
70 et al. 2009; Leto et al. 2009; Sudarov et al. 2011). Terminal commitment into BC or SC phenotypes

71 remains plastic until the final laminar position is reached, suggesting that MLI precursor fates are

72 specified by positional cues (Leto et al. 2009).

In this study, we combined large-scale labeling and reconstruction of MLIs with clustering and

74 pseudotime trajectory inference methods to define the diversity of MLI subtypes at maturity, and to

75 chart the diversification of post-mitotic precursors during development. We devised a novel single

$76 \mathrm{MLI}$ anatomy platform that: 1) employs genetic tools to sparsely label MLIs across the repertoire of

77 phenotypes, birthdates and laminar positions through development; 2) reconstructs dendritic and

78 axonal morphologies of MLIs; 3) quantifies phenotypes using morphometric parameters that describe

79 dendritic, axonal, and somatic attributes of each cell; and 4) applies unsupervised clustering and

80 pseudotime ordering algorithms to parse morphological trends among a seemingly heterogeneous

81 neuronal population (Figure 1A). We compiled a dataset of 79 complete reconstructions of mature

82 MLIs, and 732 axonal reconstructions that capture MLIs across development. Our analyses reveal

83 that mature MLIs sort into two discrete subtypes, one of which bears canonical descriptions of

84 basket cells and the other which displays a continuum of basket-stellate cell phenotypes. In a novel

85 approach to quantify diversity based on morphogenesis, we adapted pseudotime algorithms recently

86 developed for single cell RNA sequencing studies to delineate the developmental segregation of MLI

87 identities. In contrast to prevailing views that MLI phenotypes segregate as a function of birth order

88 and laminar position, we provide evidence that basket and stellate cell fates emerge early in their

89 developmental trajectory. Our study also presents a novel framework that is broadly applicable for

90 defining the trajectory of neuronal diversification using morphological information.

\section{Results}

92 A genetic platform for large-scale labeling of MLls reveals diverse morphologies

93 To systematically define MLI diversity, we sought to acquire a high-quality inventory of morphological

94 reconstructions that captures the repertoire of mature MLIs across molecular layer (ML) positions. We

95 devised a single neuron anatomy pipeline in which sparse labeling of MLIs is achieved by injection

96 of Gad2-ires-Cre mice with AAV vectors encoding multicolor fluorophores (Cai et al. 2013; Taniguchi

97 et al. 2011). As Gad2-Cre is expressed by other GABAergic populations in the cerebellum, selective

98 MLI labeling was achieved by injecting AAVs at later postnatal stages. Although AAV delivery at P0

99 resulted in the nearly exclusive labeling of Purkinje cells, injections at P5-7 or P10-14 led to enriched

100 labeling of MLIs residing in the deep or superficial ML, respectively (Figure 1B-D). Images of single

$101 \mathrm{MLIs}$ with complete axonal and dendritic arbors were acquired by confocal microscopy and were 102 reconstructed for feature extraction and analyses. We compiled 79 reconstructions of mature MLIs 103 with somas located across laminar positions (Figure 1D), and extracted 27 features that quantify 104 their dendrites, axon, soma, and location (Supplementary Table 1). Together, our dataset contains 105 high-resolution anatomical information of MLIs sampled across ML locations.

106 We began with manual expert classification of the reconstructions to determine the proportion 

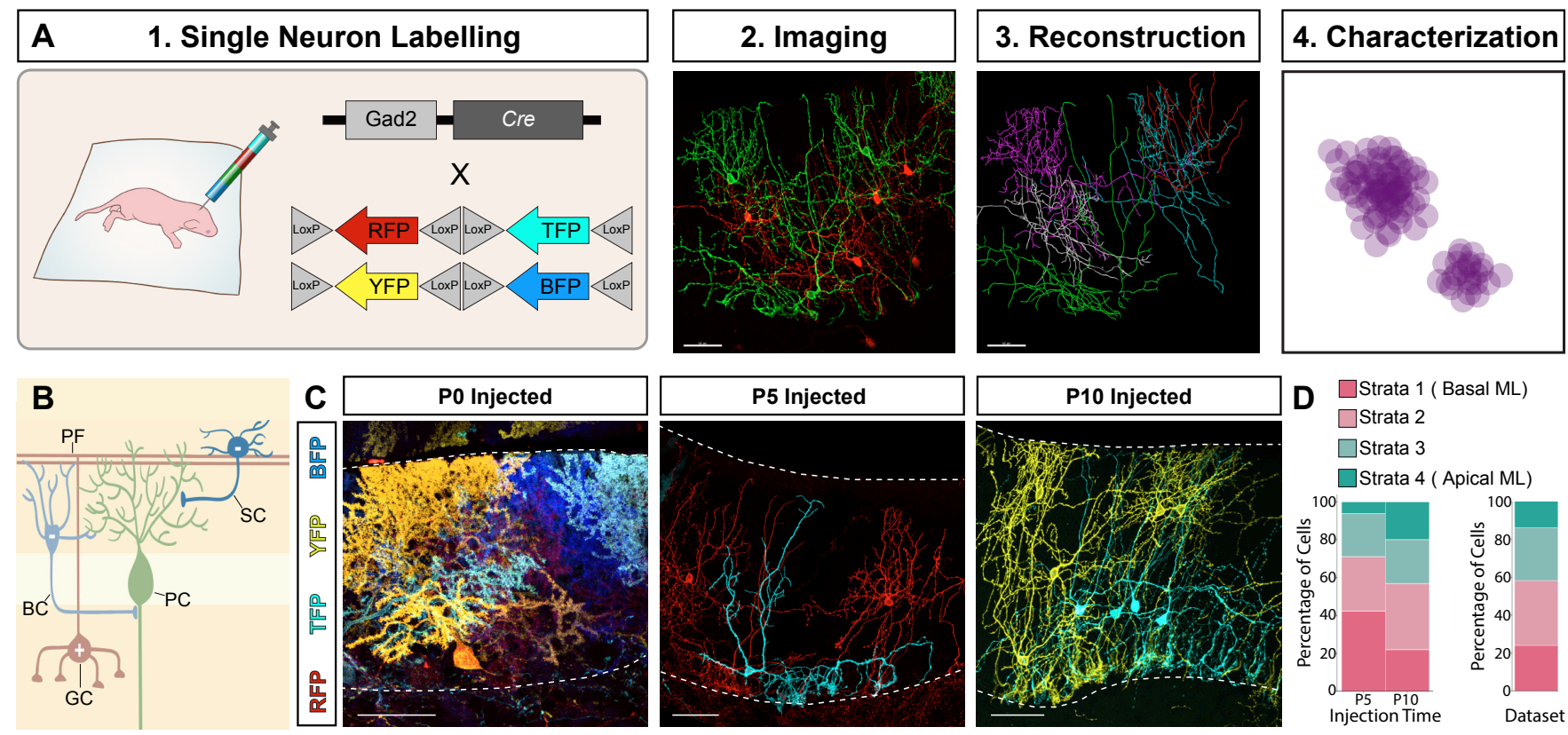

Figure 1. Platform for morphological studies of molecular layer interneurons. (A) Schematic summarizing pipeline for morphological characterizations: 1) single neuron viral labeling with Cre-dependent Brainbow AAVs encoding multiple fluorescent proteins (RFP, YFP, BFP, TFP); 2) confocal imaging; 3) semi-automated digital reconstruction and morphometric quantifications using Imaris; 4) multivariate analyses. Scale bars are $50 \mathrm{um}$. (B) Schematic of basket cells (BC, light blue) and stellate cells (SC, dark blue) that reside in the lower and upper molecular layer (ML), respectively. Canonical BC axons target the Purkinje cell (PC) soma and axon initial segment (AIS). SC axons target the PC dendrites. (C) Images of mature cerebellar cortex shows fluorescent labeling of Gad2-Cre positive populations by AAV delivery at different postnatal ages. AAV injection at P0 predominantly labels PCs; P5, labels lower MLIs; P10, enriches labelling of upper MLIs. The upper and lower bounds of the ML are outlined by dashed lines. Scale bars are 50 um. (D) Left: percent laminar distribution of singly labeled MLIs in our Brainbow AAV injection scheme, based on their laminar distribution defined by soma location in one of four ML strata. Right: Percent laminar distribution of the 79 mature single MLI reconstructions in our dataset.

107 of MLIs in our dataset that conform to canonical basket cell (BC) or stellate cell (SC) characteristics

108 (Sultan and Bower 1998; Amat et al. 2017). Canonical BCs were identified by soma location in

109 the basal third of the ML, a fan-shaped dendritic arbor that reaches the apical ML, and a long

110 axonal projection which forms multiple basket terminals (16 of 79 cells, Figure 2A, B). Cells with

111 stereotypical SC features were identified by their location in the superficial ML and their highly

112 branched, radial dendritic arbors (25 of 79 cells, Figure 2A, C). Surprisingly, nearly half of the MLIs

113 in our dataset do not fit into either category (non-canonical MLIs, 38 of 79 cells). They include MLIs

114 located in the middle of the ML displaying graded mixtures of BC and SC morphologies that vary

115 with laminar position (Figure 2D), as described previously (Sultan and Bower 1998; Rieubland et al.

116 2014). Additionally, we uncovered MLIs with morphologies that do not correlate with ML depth. For

117 example, MLIs lacking basket formations but resembling SCs were observed in the deeper third of

118 the ML (Figure 2E), and basket-forming cells were detected in the superficial ML (Figure 2F). Thus,

119 qualitative assessments reveal extensive heterogeneity in axonal and dendritic morphologies

120 among MLIs located across laminar positions.

\section{MLls form two discrete cell types that are distinguished by axonal signatures}

122 To test if the repertoire of MLIs can be distinguished into subtypes, we applied unsupervised 

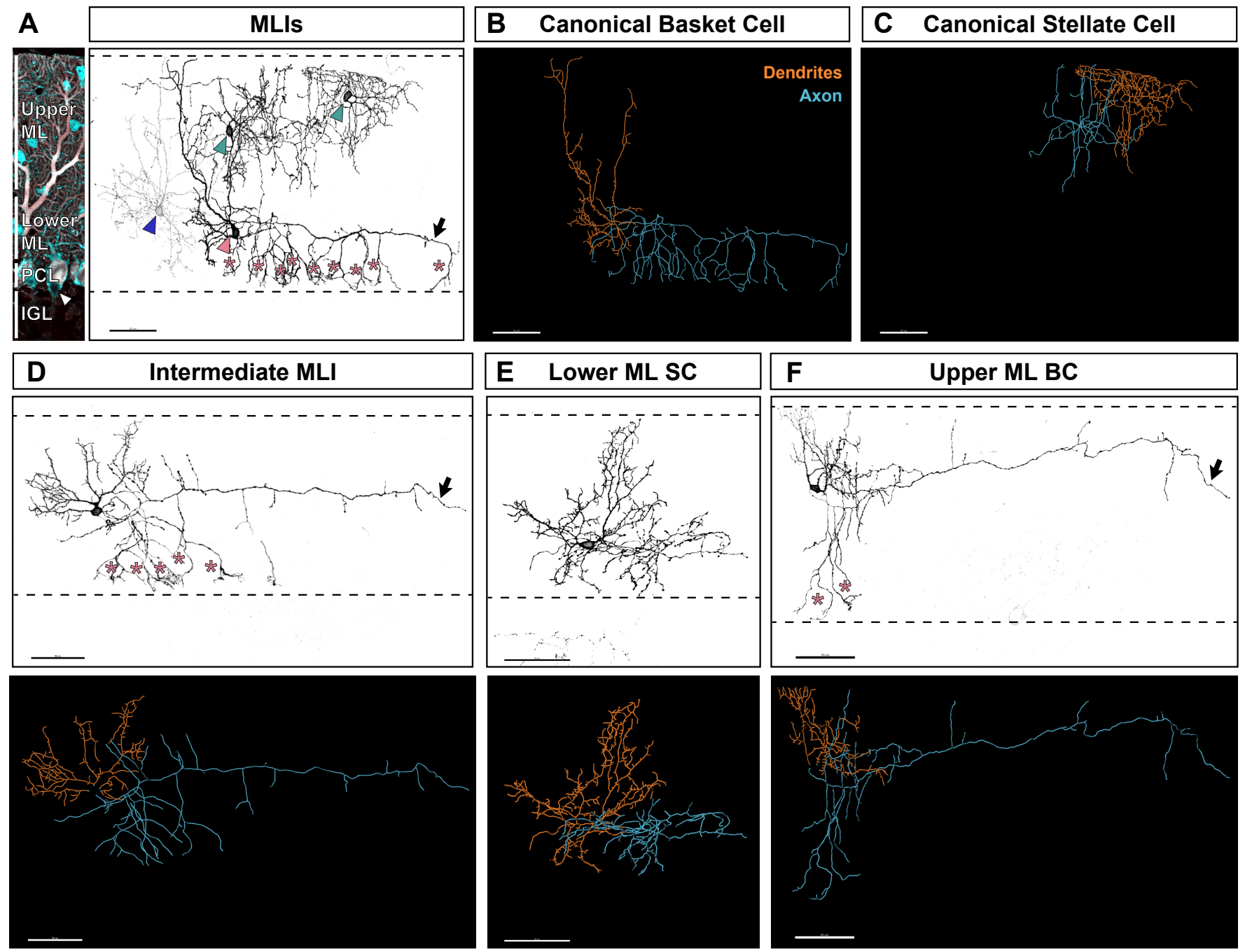

Figure 2. Morphological heterogeneity among MLIs. (A) Left: Immunostaining of MLIs (Parvalbumin, cyan) and PCs (co-labeled by Parvalbumin and Calbindin, cyan and red, respectively) show the ML space in which MLIs reside. PC somata are enveloped by BC axons (white arrowhead). Right: Inverted image of four fluorescently-labelled MLIs. The lower MLI (pink arrowhead) has canonical BC morphologies, including axonal basket terminals around the PC somata (asterisks). The two upper MLIs (teal arrowhead) have canonical SC morphologies and reside within the upper ML. The faintly labelled MLI (blue arrowhead) has SC morphologies but reside within the lower ML. (B) Reconstruction of canonical BC from panel A, with complete dendritic arbor traced in orange, and complete axonal arbor traced in blue. (C) Reconstruction of canonical SC from panel A (cell on far right). (D-F) Representative images of MLIs with mixtures of BC and SC characteristics. Top: inverted fluorescence image; Bottom: reconstruction with dendritic (orange) and axonal (blue) traces. (D) MLI located in the middle ML with SC dendritic features, and a long horizontal axon (arrow) with descending basket collaterals (asterisks). (E) MLI located in the lower ML with SC-like dendritic and axonal arbors. (F) MLI located in the upper ML with a long horizontal axon (arrow) and two descending axon collaterals with basket formations around PC somas (asterisks). All scale bars are $50 \mu \mathrm{m}$.

123 clustering methods to analyze the dataset of single MLI morphometric properties. We first 124 performed hierarchical clustering which rendered two major clades (Figure $3 \mathrm{~A}$ ). We inspected 125 each reconstruction to relate the hierarchical ordering to morphological phenotypes. The first clade 126 contained basket cells displaying canonical features and corresponding to the manually curated 127 BCs ( $n=19$ cells; Figure 3A-C). By contrast, the second clade was larger, comprising canonical SCs 128 and the remaining non-canonical MLIs ( $n=60$ cells; Figure 3A). Notably, cells in this SC-like clade 129 (hereafter SCs) were ordered into subclades that followed a linear progression in morphological 130 characteristics. Visually, we discerned four subdivisions that reflected similarities in shapes and 

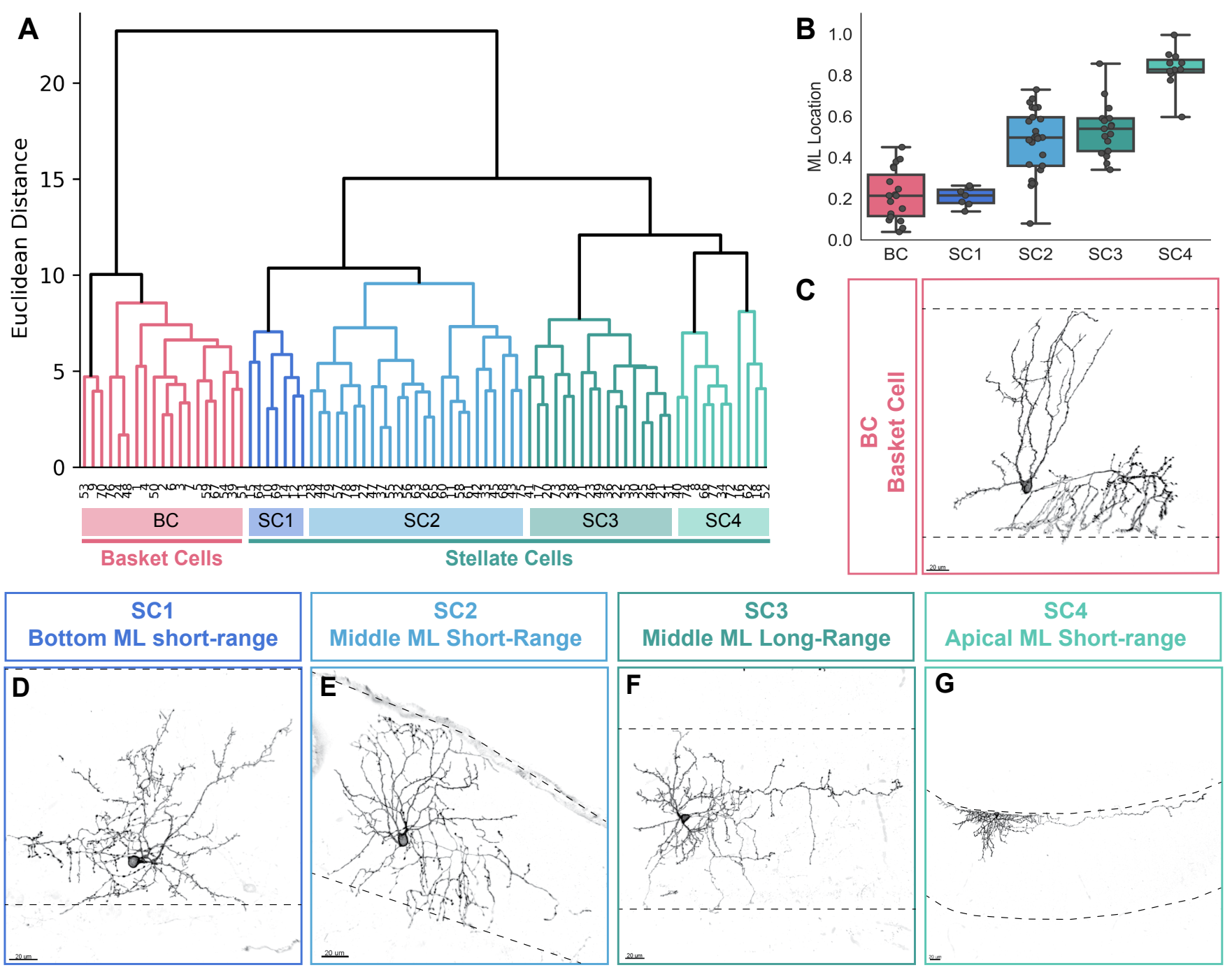

Figure 3. Hierarchical clustering of mature MLI morphologies unmasks two distinct morphological groups. (A) Hierarchical clustering dendrogram. The BC cluster (pink) is separate from the SC cluster (blue-greens). Within the broad SC cluster, the dendrogram ordered MLIs based on shared morphological characteristics and was manually curated into four subclades based on expert assessment of similarities. (B) ML Location of MLIs sorted into designated subclades. (C-G) Individual MLI examples from the SC subclades (SC1-SC4). Dashed lines denote the apical and basal extremities of the ML. Scale bars are $20 \mu \mathrm{m}$.

131 ML locations: SC1, stellate cells with short-range axons located in the bottom ML (Figure 3B,

132 D; non-basket forming); SC2, short-range stellate cells in the middle and upper ML (Figure 3B,

$133 \mathrm{E})$; SC3, stellate cells in the middle and upper ML with long-range axons, some of which give

134 rise to 1-3 basket collaterals (Figure 3B, F); and SC4, long-range stellate cells constrained to the

135 apical ML (Figure 3B, G). By statistical methods however, the optimal number of clusters for the

136 total MLI dataset were identified as two by silhouette analysis and to three by the elbow method

137 (Supplementary Figure S1; Rousseeuw 1987; Thorndike 1953). We reasoned that further divisions

138 may be ambiguous due to the limitations of these tests or due to heterogeneity among the SC

139 population.

140 To test if MLIs could be further parsed into subtypes by other clustering methods, we applied

141 the Uniform Manifold Approximation and Projection algorithm to the morphometric dataset (UMAP;

142 Becht et al. 2018; Mclnnes et al. 2018). UMAP is a dimensionality reduction algorithm that projects 

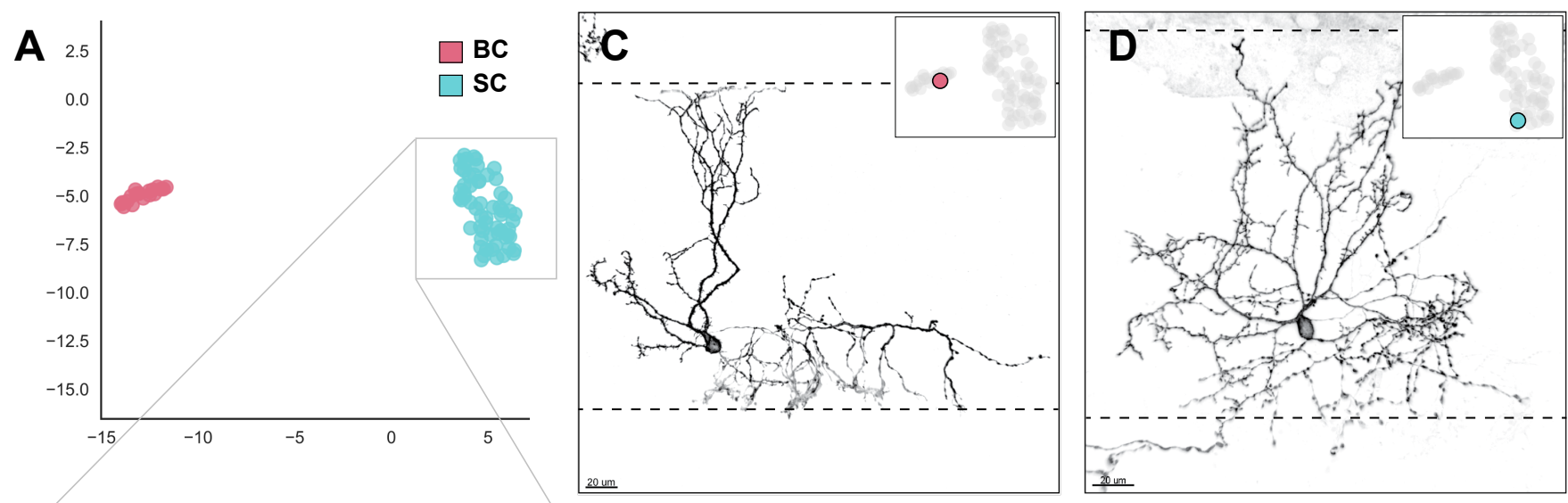

B
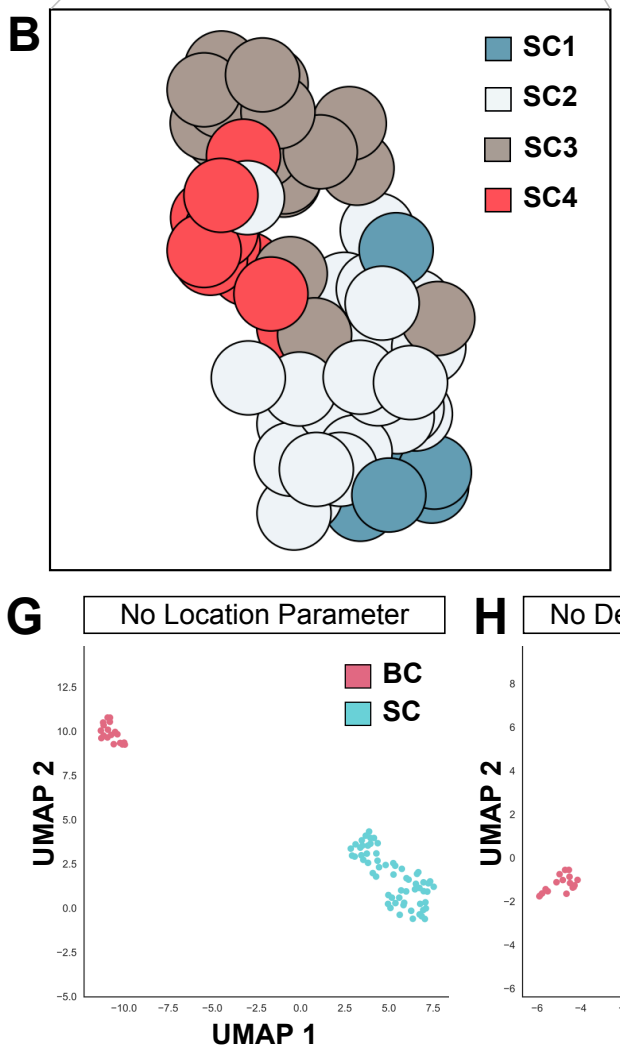

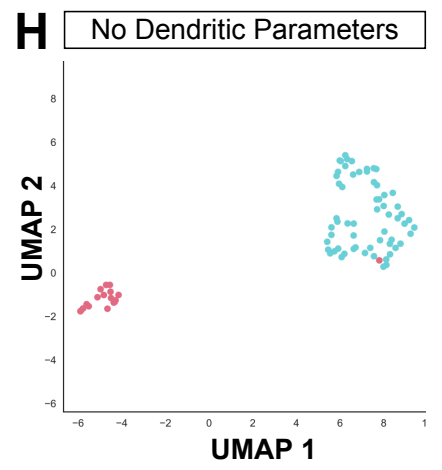

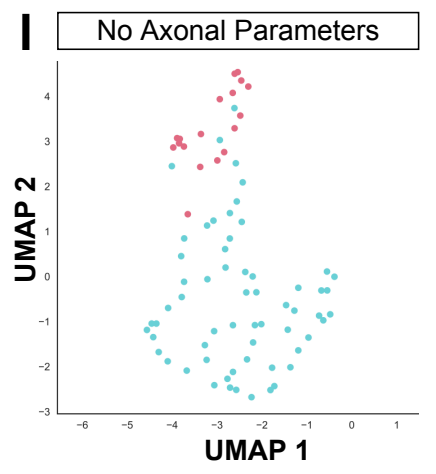

Figure 4. UMAP of mature MLI morphologies uncovers two distinct subtypes. (A) UMAP projection of 79 mature MLI reconstructions in our dataset. Colors indicate BCs (pink) and SCs (teal). (B) Projection of SC subclade identity from hierarchical clustering results projected onto the SC cluster. (C-F) Individual examples of MLIs validate the morphological correspondence between MLI clusters. (C) MLIs from the BC cluster (inset) display canonical BC morphologies. Basal and apical limits of the $\mathrm{ML}$ are outlined by dashed lines. (D) An MLI at one pole of the SC cluster (inset) displays short-range axonal morphology and resides within the basal ML. (E) An MLI at the opposite end (inset) displays long-range axonal morphology, while a cell at a neighboring datapoint (F) has a similar form but extends descending axon collaterals to PC somata (pink asterisk). (G-J) UMAP projections following recursive elimination of morphological features describing $M L$ location $(\mathbf{G})$, dendrites $(H)$, or axons (I). Axonal information was necessary (I) and sufficient $(\mathrm{J})$ for UMAP-dependent MLI clustering. Scale bars are $20 \mu \mathrm{m}$ for $\mathrm{C}$, D and $\mathrm{F}$, and $40 \mu \mathrm{m}$ for $\mathrm{E}$.

143 each MLI reconstruction onto a two-dimensional space based on local similarities with other MLIs.

144 UMAP rendered two clusters and reproduced the BC and SC division, similar to hierarchical

145 clustering (BC cluster, $n=18$ cells; SC cluster, $n=61$ cells; Figure 4A-F). Although the SC cluster is

146 broad and contains morphologically heterogeneous cells, the cells largely co-distributed within the

147 curated phenotypic subclades from hierarchical clustering (Figure 4B, E, F). Moreover, distantly 
148 projected cells exhibited different phenotypes, such as the projection of lower ML short-range SCs 149 at one end of the cluster and upper ML long-range SCs at the other (Figure 4 D,E). A similar division 150 between the BC and SCs was produced with the t-Distributed Stochastic Neighbor Embedding 151 (t-SNE) algorithm (Supplementary Figure S2). Taken together, these analyses robustly divide MLIs 152 into two discrete populations, the BC and SC types, but the SC population exhibits considerable 153 heterogeneity.

154 We next examined the morphological within-class heterogeneity present within the SC 155 population. It was previously proposed that MLIs model a single continuously varying population 156 based on ML laminar positioning (Rieubland et al. 2014; Sultan and Bower 1998). We wondered 157 if this heterogeneity may be accounted for through variations within the SC cluster alone. To test 158 this possibility, we performed regression analysis on all morphological features as a function of ML 159 location, first using all 79 reconstructions in our dataset (Supplementary Figure S3). We found 8 160 parameters which showed significant positive or negative correlation with soma position within the 161 ML, including dendritic complexity, as previously described (Rieubland et al. 2014). We performed 162 an additional round of regression analysis for each of these 8 parameters, using only the 61 SCs in 163 our dataset. We found that for 4 of these parameters, the correlation remained even after removal 164 of all BCs from the analysis (Supplementary Figure S3). The remaining 4 parameters corresponded 165 to features where the BC population added a significant skew to the correlations graph, and where 166 the entire population had not modeled continuous variation in the first place. We further confirmed that all 4 parameters followed a largely normal distribution within the SC type, with no presence of bimodality (Supplementary Figure S4). Together, our results suggest that the significant variation among SCs denotes continuous variation, similar to what was proposed previously for the entire MLI population.

Finally, to define the features most informative for MLI classification, we analyzed the dataset using recursive feature elimination, in which single or groups of morphological features were removed. The BC/SC division remained following elimination of all dendritic features and, 174 surprisingly, elimination of soma location (Figure 4G, H; Supplementary Figure S2). By contrast, BC 175 and SC clustering was lost upon removal of all axonal features (Figure 4I). To test whether axonal 176 phenotypes alone are sufficient for clustering, we restricted UMAP analyses to six parameters that 177 describe axonal morphology. Interestingly, the BC and SC clusters were recapitulated with all but one 178 cell correctly sorted (Figure 4J). Therefore, despite the anatomical complexity and heterogeneity, 179 axonal information is necessary (Figure 4I) and sufficient (Figure 4J) for the classification of MLIs 180 into BC or SC subtypes. We conclude that MLls form two discrete cell types. While our results 181 support the classical BC and SC division, the significant heterogeneity present within the SC group 182 also explains previous observations of continuous variation that was the basis for the one MLI 183 population model (Sultan and Bower 1998). 
A
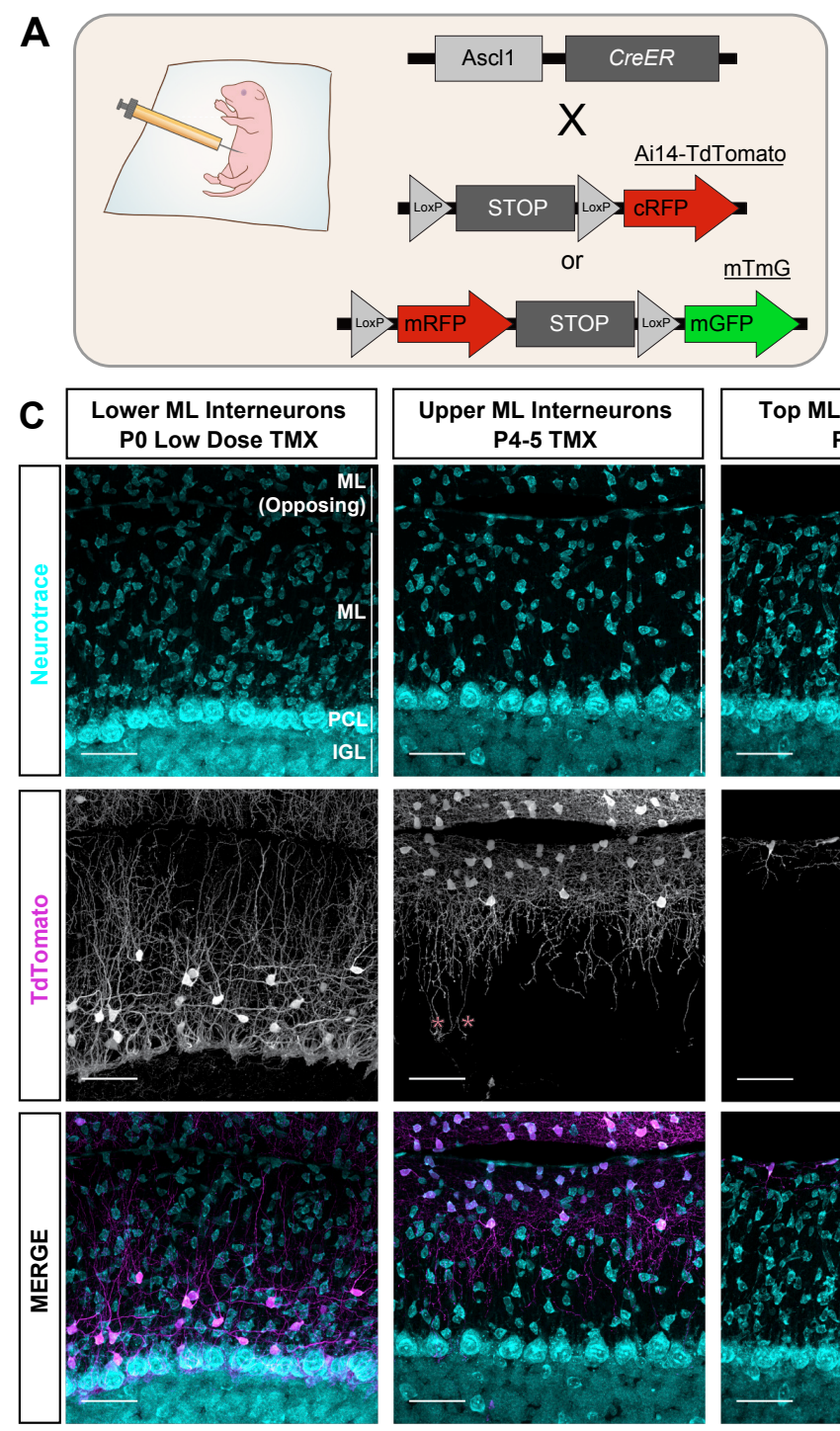
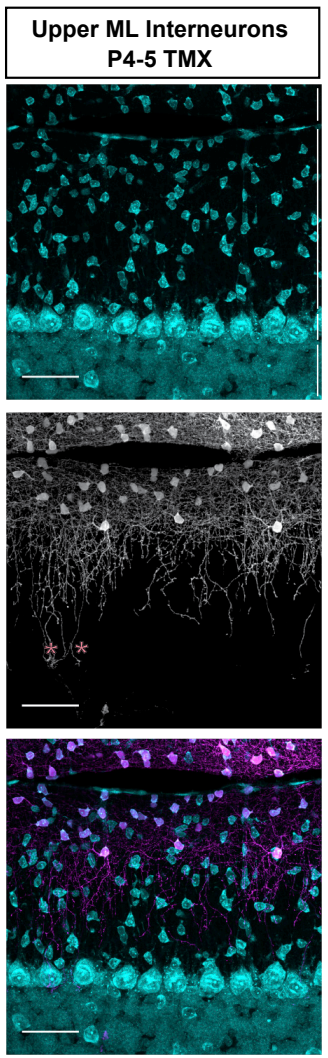

B

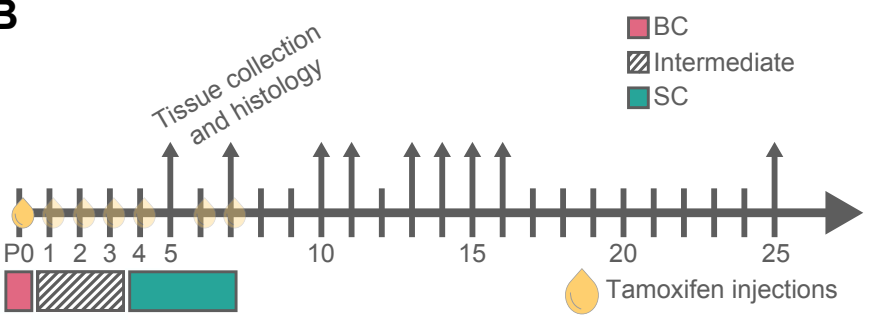

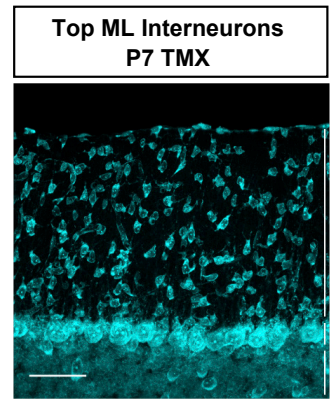
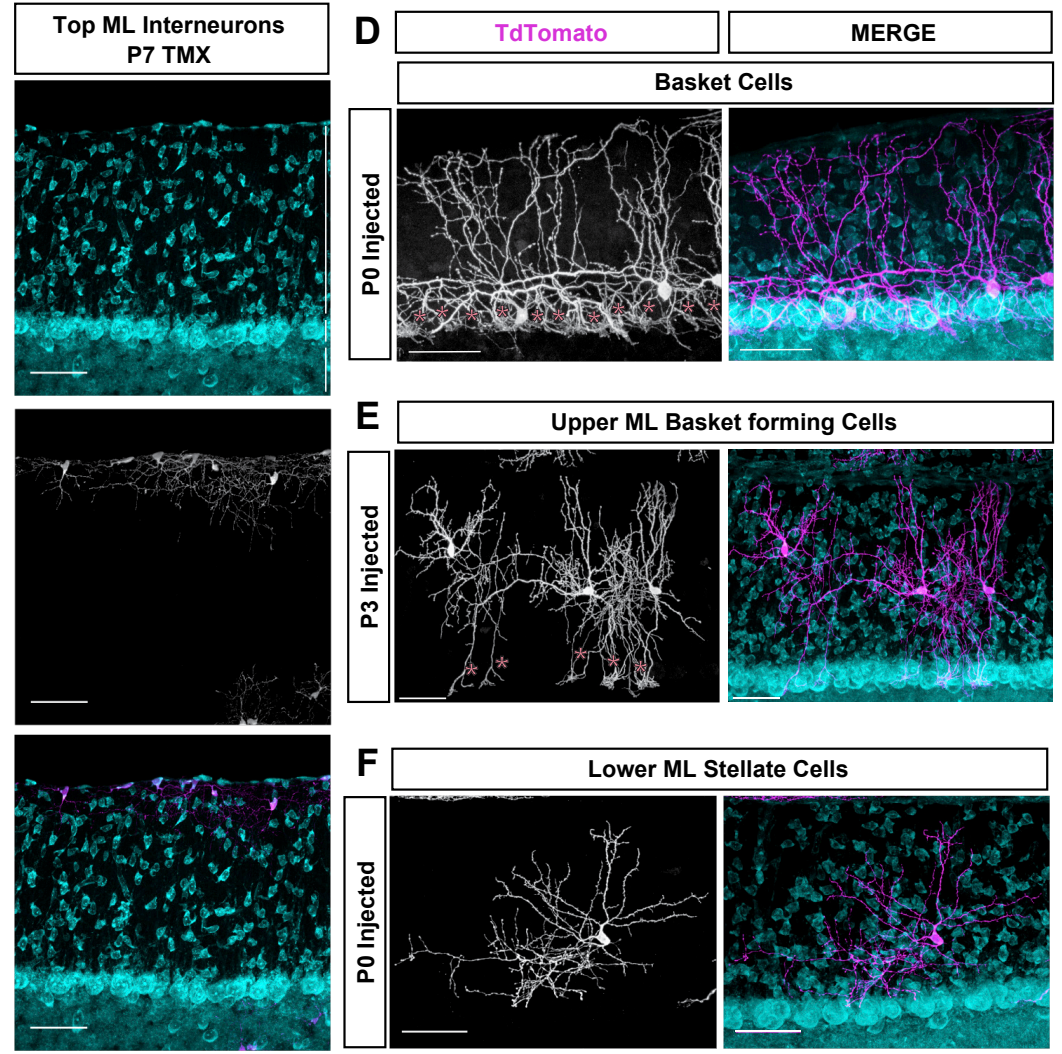
Figure 5. Birthdate-dependent targeting of MLI subpopulations by Ascl1-CreER. (A) MLI labeling strategy by tamoxifen (TMX)
inducible Ascl1-CreER and fluorescent Cre reporters, Ai14-TdTomato (cytosolic) or mTmG (membrane-targeted). (B) Schematic for TMX-induced subtype-enriched MLI labelling. Postnatal timepoints of single dose TMX injections are depicted by oil droplets. Collection timepoints are shown by upwards arrows. Low dose induction at P0 predominantly labels BC subpopulations, P2-P3 induction labels intermediate MLIs, P4-7 injections label SC cells in the upper ML. (C) Confocal images of Ascl1-CreER; Ai14 cerebellar cortex at P25 shows laminar restriction of MLI labeling (TdTomato, greyscale or magenta; Neurotrace, cyan) following TMX induction at P0, P4, or P7. (D) Example of P0-induced BCs in the lower ML. (E) Example of P3-induced SCs in the upper ML with descending basket terminals. Pink asterisks denote basket formations enveloping PC soma in D, E. (F) Example of P0-induced SC in the lower ML. Representative images were taken at P25. Scale bars are $50 \mu \mathrm{m}$.

\section{Genetic fate mapping of MLIs reveals a population of early-born stellate cells}

185 Having established the divergence of MLIs into two subtypes on the basis of axonal information,

186 we wondered whether the emergence of MLI identities could be traced during development based

187 on axon morphologies. The differentiation of MLIs is largely uncharacterized and there are no

188 established molecular markers to distinguish BCs and SCs at maturity nor during development

189 (Sotelo 2015; Schilling and Oberdick 2009). Therefore, we reasoned that morphological mapping

190 could reveal time points and locations that influence MLI diversification into basket and stellate

191 cell phenotypes. To date, fate mapping and heterochronic cell transplantation studies have led 
192 to a series of observations that together suggest that MLI identities are determined by birth order 193 and laminar location. First, MLIs are generated from a shared progenitor pool but laminar specific 194 phenotypes result from the sequential generation and the inside-out settling of precursors within 195 the cerebellar cortex (Altman and Bayer 1997; Leto et al. 2009; Sudarov et al. 2011; Zhang and 196 Goldman 1996b). Second, terminal commitment into BC or SC identities is plastic until the final 197 laminar position is reached (Leto et al. 2009). According to this model, early-born MLls that first 198 populate the lower ML adopt BC identities, while later-born MLIs that fill the upper ML become 199 SCs. To directly test this model, we devised a genetic fate mapping strategy to label single MLIs 200 born on different days and trace their locations and patterns of axon morphogenesis. We used 201 the tamoxifen-inducible Ascl1-CreER line to activate Cre in MLI progenitors at the time of terminal 202 division (Sudarov et al. 2011; Brown et al. 2019). Consistent with previous studies, tamoxifen 203 (TMX) injection of Ascl1-CreER; Ai4 flox-STOP-TdTomato animals at P0 enriched for the labelling of BCs that innervate the lower ML, while delivery at P4 and P7 marked SC-like cells restricted to the upper ML (Figure 5A-C).

To determine the relationship between MLI birthdate and terminal fate, we characterized the mature morphologies and laminar positions of MLIs labelled by TMX injections on different days. Additionally, we aimed to relate SC subclade identities established from hierarchical clustering analyses to their birth order (Figure 3). CreER induction at PO labelled BCs in the deep ML (Figure 5D), while induction at P2-3 predominantly marked MLIs with mixed BC-SC phenotypes, consistent with the middle and upper ML basket forming cells of SC3 (Figure 5E). The majority of MLIs labelled at P4-5 were non-basket forming short- and long-range SCs in the superficial ML, typical of SC2 and SC3 (Figure 5C). P7 inductions labelled apical ML constrained SC4 cells nearly exclusively (Figure 5C). Intriguingly, we observed that SC1s which reside within the lower ML were marked at P0, and to a smaller extent at P1 (Figure 5F). However, they were not detected following CreER induction at P3 and onwards, suggesting that the lower ML SC1s are early-born cells within the MLI

217 lineage. These findings support one aspect of the current model in that the inside-out stratification 218 of MLIs within the molecular layer is temporally related to birth order. However, they also suggest 219 the existence of an early-born SC subpopulation which shares a similar temporal origin and laminar 220 fate to BCs but adopts a distinct, non-basket forming phenotype. Our findings therefore indicate that BC and SC phenotypes are not strictly separated by temporal origin or laminar positioning.

\section{MLI axon morphogenesis can be ordered along a pseudo-temporal maturity}

\section{3 gradient}

224 The marking of early-born MLIs that elaborate distinct phenotypes in the lower ML suggests the 225 divergence of BC and SC fates during early postnatal development. We hypothesized that if MLIs 226 diverge into two early-born BC and SC precursors, the subpopulations would progress through 227 distinct developmental trajectories. Alternatively, if MLI subtype differentiation is dependent on 228 positional cues, such as those present at the site of integration, then MLI precursors might share 
229 a similar progression until they settle and elaborate subtype-specific axonal arbors. To distinguish between these possibilities, we sought to define the divergence of $\mathrm{BC}$ and $\mathrm{SC}$ identities by comparing patterns of migration and axon morphogenesis. To incorporate developmental data spanning all 232 stages of development, we adapted a pseudo-temporal ordering approach to align snapshots of 233 single neuron morphologies along a continuous progression of maturation. Pseudotime trajectory inference was recently developed for analyses of single cell transcriptomics datasets, where cells dissociated from developing tissue at a fixed time contain a spectrum of cellular states due to the asynchronous nature of development (Figure 6A; Setty et al. 2019; Trapnell et al. 2014; Saelens et al. 2019; Serra et al. 2019). Thus, computational alignment of single cell data along a continuous maturity gradient enables a model for the progression and bifurcations of cellular states (Trapnell et al. 2014). In a similar vein, we applied pseudotime modelling to infer the continuous trajectories of MLI development using high dimensional morphometric data of single neurons (Figure 6A).

We acquired a large dataset of developing MLI morphologies marked by low-dosage TMX injections and collected over a series of timepoints spanning MLI morphogenesis (P5 - P21) (Figure 5B). Axonal arbors of $732 \mathrm{MLIs}$ were reconstructed and quantified for 28 morphometric parameters describing their axon, soma and location. Each reconstruction was annotated for the cell's inferred terminal fate (early-born, BC or SC1s: P0 TMX injected; late-born, SCs: P3-7 TMX injected) and age (days post TMX injection, hereafter DPI). In parallel, each cell was manually binned into one of four maturation stages according to a qualitative assessment of dendritic arborization and soma shape (Supplementary Figure S5). This scheme served as an expert-directed ordering to validate the performance of pseudotime algorithms in aligning axonal reconstructions along a maturation trajectory.

We first evaluated the suitability of pseudotime algorithms for modeling axonogenesis and if so, to determine whether separate BC- and SC-fated trajectories can be distinguished. We began with analysis of the early-born MLIs (P0 TMX, 423 cells) using Palantir, a diffusion map-based pseudotime algorithm (Setty et al. 2019; Coifman et al. 2005). Palantir rendered a trajectory that followed a linear progression, as confirmed through both the default and expertdirected pseudo-timelines (Figure 6B; Supplementary Figure S6, 7). To evaluate the accuracy of the manifold, we clustered the trajectory into eight developmental states using PhenoGraph (Levine et al. 2015), which computationally partitions high-dimensional datasets into phenotypically meaningful subpopulations (Figure 6C). Inspection of clusters along the trajectory revealed a robustly ordered pseudotime ordering of canonical BC axon morphogenesis, in clusters 1, 2, 3, 4, 6 , and 8 (Figure $6 \mathrm{C}-\mathrm{I}$ ). Cluster 1 marked the beginning of the $\mathrm{BC}$ trajectory, where cells displayed the simplest morphologies consisting of a single, short axonal extension (Figure 6D). The end of the trajectory was represented by cluster 8 , which contained cells with the most complex axonal arbors, and increasing elaborations of basket terminals (Figure 6l). Importantly, the intervening clusters uncovered previously unknown trends during BC morphogenesis, in addition to known 266 characteristics. Cluster 1 and 2 cells possessed a leading process and exhibited immature soma 


\section{A Ordered by real time}
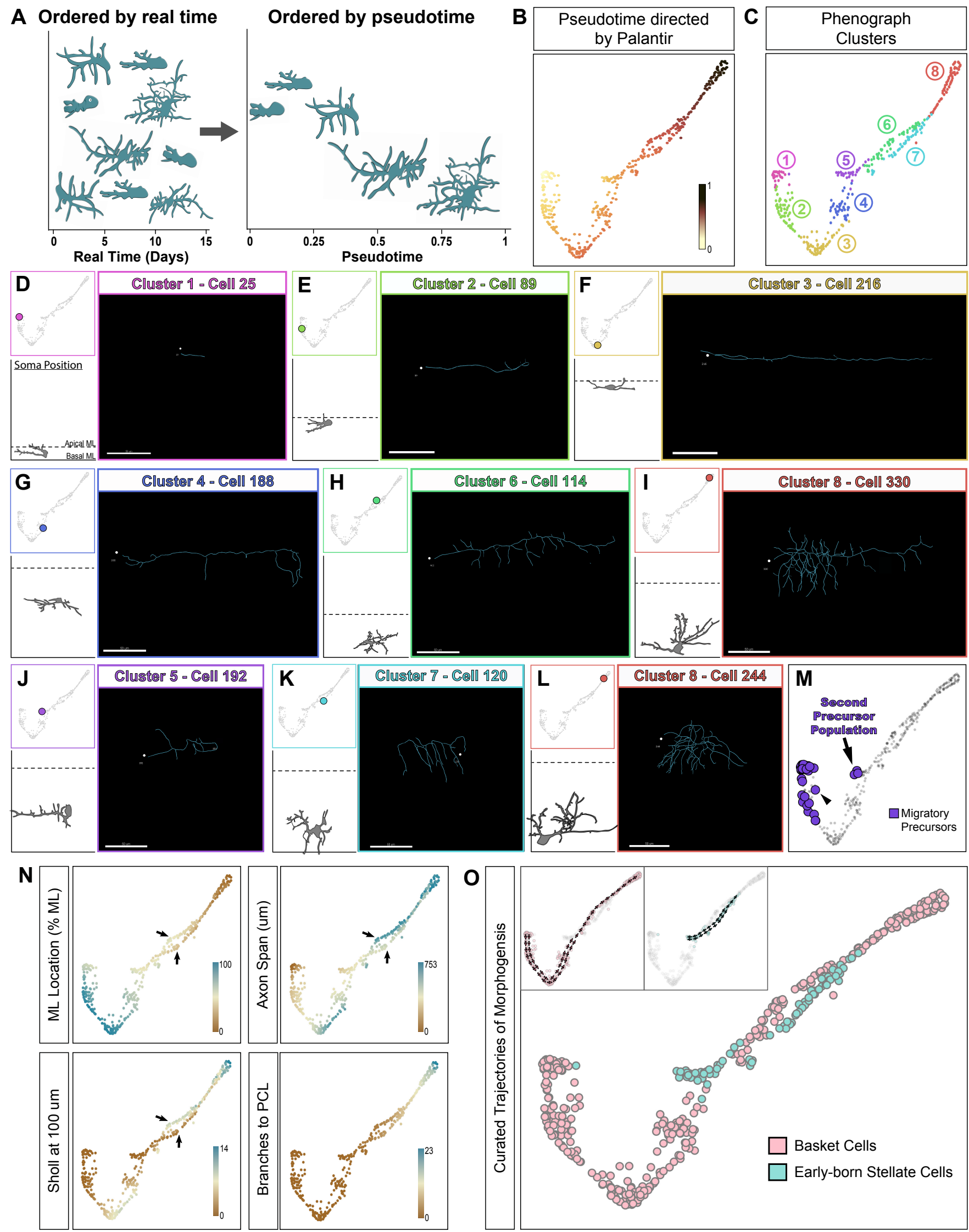

Figure 6. Pseudotime ordering of early-born MLIs based on quantitative analyses of axonal development reveals two lineage trajectories. (A) Schematic showing the basis of pseudotime trajectory inference methods. If ordered by real time (i.e. by animal age, left), progressions of neuronal maturation are difficult to ascertain due to variability of developmental stages. Pseudotime 
orders snapshots of single developing neurons based on morphological progression rather than animal age (right). (B) Palantirgenerated pseudotime ordering of early-born MLIs (P0 TMX injection - collected P5 - P25). Each datapoint represents one reconstruction from the dataset of developing MLI axon morphologies. (C) Phenograph-generated division of the axonal dataset into eight clusters that reflect different morphological stages. (D-M) Examples of single MLI morphologies plotted within each cluster 1-8. Top left: Position of cell in pseudotime trajectory. Bottom left: Camera Lucida illustration of cell soma and dendrites, positioned to scale by ML location, to validate progressive maturation of cells. Right: Axonal reconstruction with soma outlined by white dot. (D-I) Representative examples along the BC trajectory of morphogenesis. (J-L) represent examples of MLIs along the early-born SC trajectory of morphogenesis. (M) Migratory precursors are plotted in two locations. In addition to the precursors for the BC population (cluster 1, black arrowhead), there is a second naive population that corresponds to early-born SCs (arrow). (N) Heatmap representation of single morphometric parameters corresponding to Palantir-ordered MLIs. Arrows highlight differences between the BC and early-born SC trajectories. BCs occupy higher positions within the ML during migration (ML location) with axons that span a greater distance along the ML (axon span and Sholl at 100um). (O) Curated pseudotime trajectories for early born MLIs. BCs are highlighted in pink, while early born SCs are highlighted in cyan. All scale bars are $50 \mu \mathrm{m}$.

267 morphologies oriented along the tangential plane of the apical ML (Figure 6D, E), consistent with 268 descriptions of tangentially migrating MLI precursors (Cameron et al. 2009; Wefers et al. 2018; 269 Wefers et al. 2017). This finding suggests that BC axonogenesis begins during neuronal migration.

270 Consistently, cells in clusters 3, 4, 6 and 8 occupied gradually decreasing laminar positions as 271 axonal morphogenesis proceeded to completion (Figure 6F-I). Together, the robust ordering of 272 BC axonogenesis demonstrates the suitability of pseudotime algorithms for aligning snapshots of 273 single neuron morphometric information and inferring the trajectory of morphogenesis.

$274 \quad$ Notably however, MLIs represented in clusters 5 and 7 do not fit the axonal progression of 275 BCs (Figure 6C). Compared to cells in clusters 3 and 6 with similar extent of dendritic maturation, 276 axonal arbors of cluster 5 and 7 cells have noticeably shorter primary axons (Figure $6 \mathrm{~J}-\llcorner$ ). Additional 277 visual curations identified a small population of migratory precursors lacking a noticeable trailing 278 process, a characteristic of immature MLIs that were also mapped to clusters 1 and 2 (Figure $6 \mathrm{M})$. This observation suggests that cluster 5 marks the beginning of a second early-born MLI trajectory (Figure 6M). We further examined this divergence by projecting individual morphometric parameters onto the manifold. The parallel tracks formed by clusters 6 and 7 differed in axonal 282 span, branching complexity and laminar location (Figure 6N; Supplementary Figure S8). A similar 283 but more substantial contrast was noted between clusters 3 and 5 (Figure 6N; Supplementary 284 Figure S8). Taken together with our previous fate mapping studies and given the short-range 285 nature of cluster 5 and 7 cells, our results support a model of two lineages of early-born MLIs that correspond to BCs and the lower ML, short-range SC1s (Figure 60)

Given the power of pseudotime inference for modeling of early-born MLI morphogenesis and uncovering de novo trajectories, we next applied Palantir to the entire suite of $732 \mathrm{MLI}$ reconstructions. Palantir produced a pseudo-timeline but the trajectory was discontiguous, with 290 alternations between immature and mature pseudotime states (Figure 7A). To test if this was due 291 to the altered alignment of cells within the manifold, or incorrect projection of pseudotime states to 292 the underlying manifold, we projected the manually annotated single cell maturation stages onto 293 the trajectory (Figure 7A and Supplementary Figure S5). Our expert-directed pseudotime approach 294 confirmed that the underlying manifold structured the cells from immature to mature states (Figure 295 7A). We further mapped the BC and SC1 lineages, as well as the late-born SCs (TMX at P4-P7; 
A

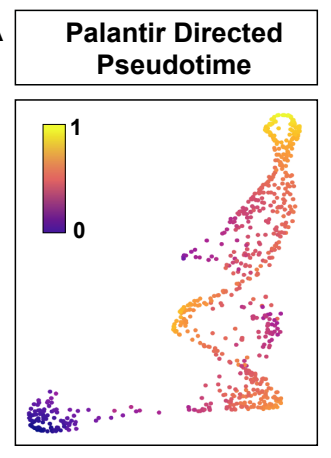

D

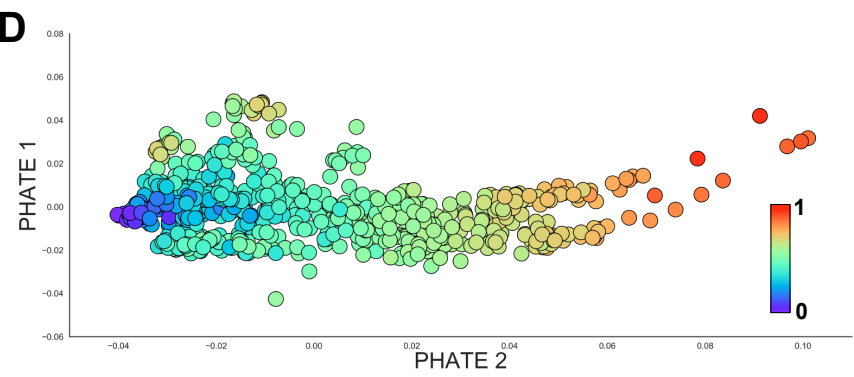

$\mathbf{F}$

F

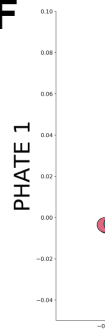

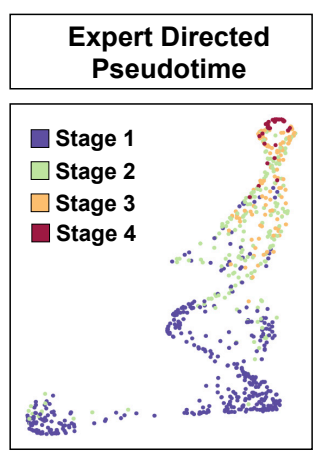

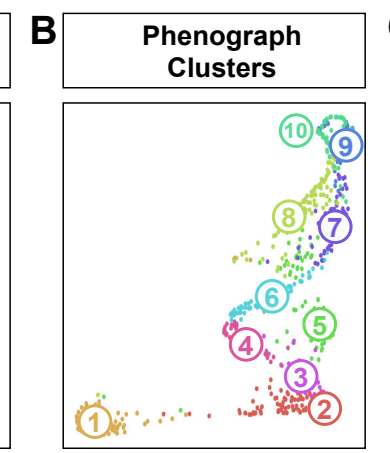

E
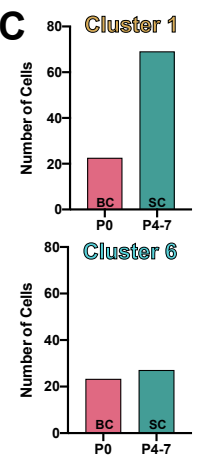
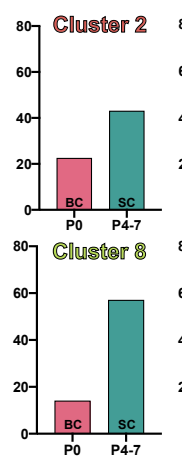
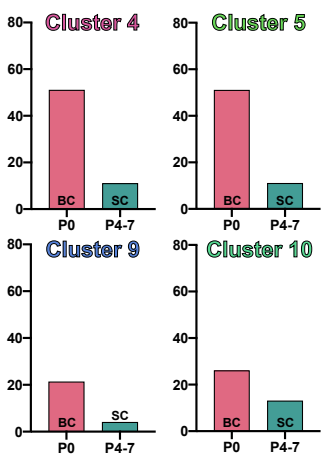

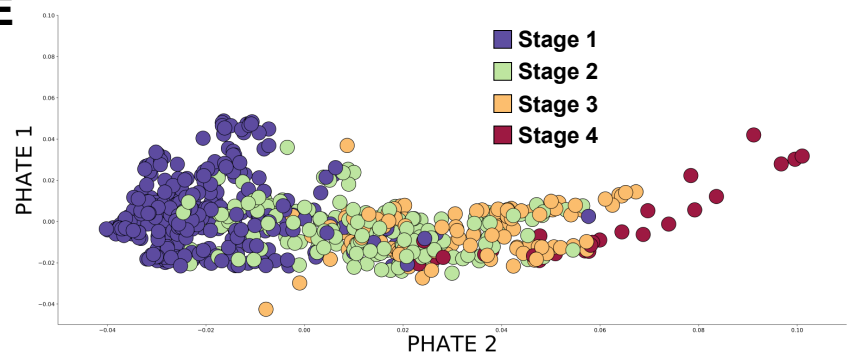

G

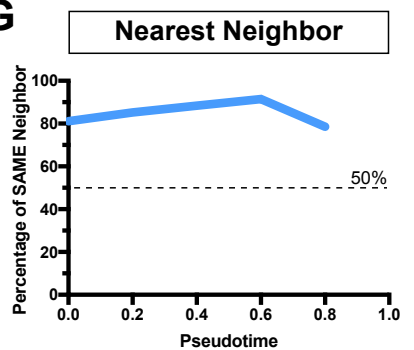

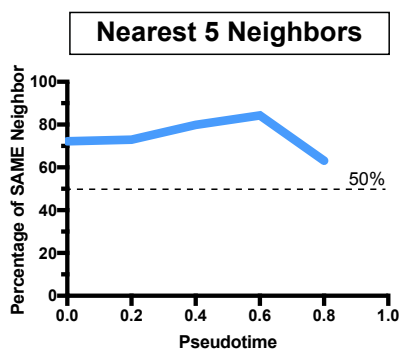

Figure 7. Emergence of MLI-type identities prior to axonogenesis. (A) Left: Palantir-generated pseudotime ordering of axonal traces from 732 developing MLIs. Right: Expert-directed pseudotime ordering of reconstructions using manual annotation of maturation based on 4 stages of dendrite progression. (B) Phenograph-generated clustering that reflects different morphological stages. (C) Number of BCs (P0 TMX injected) or SCs (P4-7 TMX injected) within each Phenograph cluster. Nearly all clusters show enrichment of BCs or SCs. (D) PHATE-generated pseudotime ordering. (E) Validation of PHATE ordering by projecting manual annotation of dendrite maturity for each MLI. (F) PHATE generated pseudotime trajectory for the entire MLI dataset, colored by BC (early born, P0 TMX; pink) or SC identity (late born, P4-P7 TMX; teal). (G) For each neuron (datapoint), the nearest 5 neighbors were obtained. Left: proportion of cells with a nearest neighbor of identical fate (i.e. BC vs SC). Right: Proportion of nearest 5 neighbors with the same identity. If $\mathrm{MLI}$ fates are established late during development, $\sim 50 \%$ of cells would be expected to have a neighbor of the same identity (dashed line). Over $80 \%$ of MLIs at pseudotime 0 have an identical neighbor, suggesting early establishment of MLI identity. The drop in percentage of identical neighbors between $0.6-0.8$ pseudotime is likely due to sparsity of mature reconstructions in our dataset, i.e. fewer data points at the right of the PHATE trajectory in (D-F).

285 Supplementary Figure S9). However, the projections were difficult to interpret as cell lineages 286 were arranged along a discontinuous pseudo-timeline and the global lineage structures were not 287 maintained. In an alternate approach, we clustered the trajectory into ten developmental states 288 and analyzed the local subtype distributions (Figure 7B; C). Nine of the ten clusters were enriched 289 for either the early-born or late-born MLI populations, regardless of pseudotime maturity, which 290 suggests clustering at the local scale (Figure 7C). The remaining cluster contained SC1 cells nearly 291 exclusively (Supplementary Figure S10). Although the Palantir-directed trajectory separated MLI 292 subtypes within pseudotime stages, the overall performance with this dataset was limited. 


\section{Trajectory inference by PHATE reveals the early emergence of BC and SC}

\section{4 identities}

295 To test if the total MLI trajectory can be resolved by other trajectory inference algorithms, we applied 296 a parallel approach using PHATE. PHATE was developed for visualization of branching data 297 structures, with preservation of both local and global similarities (Moon et al. 2019). The PHATE298 generated trajectory ordered the MLI reconstructions along a linear arrangement that reflected a 299 pseudo-temporal gradient (Figure 7D), and was confirmed by our expert-directed staging metric 300 (Figure 7E). Projection of the early- and late-born MLIs revealed that the two populations largely 301 segregated along the trajectory from the beginning of pseudotime (Figure 7F). To quantify this 302 observation, we performed nearest neighbor analyses following pseudotime binning (Figure 7G). 303 We reasoned that if the terminal fates of MLI precursors remain undetermined at the beginning of 304 axonogenesis, the identity of a cell's nearest neighbor should follow a largely random distribution. 305 Correspondingly, roughly $50 \%$ of early pseudotime cells should possess a nearest neighbor of the 306 same prospective terminal fate. Alternatively, a positive or negative deviation from $50 \%$ infers an 307 early bias of MLI type identities. In support of the latter, $80 \%$ of MLIs at the origin of pseudotime 308 possessed a nearest neighbor with an identical terminal fate (Figure 7G). We further confirmed this 309 finding by extending the analysis to the nearest 5 neighbors (Figure 7G). Taken together, these 310 results provide evidence for an early divergence model for MLI type identities. Our data support 311 a scenario where migratory MLI precursors at the beginning of axonogenesis are biased towards 312 their terminal fates.

313 To determine whether divergent axonogenesis patterns are visually discernible, we 314 compared pseudotime to real time morphologies within the in situ tissue space. At 5-6 days post 315 injection (DPI), we observed tangentially migrating BCs and SCs that bear a long axonal process 316 (Figure 8A, B). Their morphologies resemble cluster 1 cells of the BC pseudotime lineage and are 317 representative of the stage 1 manual classifications (Supplementary Figure S5). By 10-11 DPI, $318 \mathrm{MLI}$ precursors displayed a mixture of developmental stages that were stratified according to the 319 inside-out layering of the ML (Figure 8C, D). Tangentially migrating stage $1 \mathrm{BCs}$ and SCs were 320 located in the apical ML, while stage 2 and 3 cells were located in progressively lower ML positions 321 corresponding to increasing axonal complexity (Figure 8C, D). The developmental gradient of cells 322 across the ML supports our pseudotime findings (see Figure 6N). By 20 DPI, nearly all labelled 323 neurons displayed axonal morphologies characteristic of mature BCs or SCs, consistent with the 324 end of pseudotime (Figure 8E, F).

325 In this visual analysis, stage $3 \mathrm{BC}$ and SC subtypes are distinguishable based on 326 differences in arbor geometry, laminar location, and axonal targeting. Quantifications of individual 327 parameters further confirmed our pseudotime findings that BCs and SCs segregate throughout 328 axon morphogenesis (Figure 8G). However, subtype differences were not visually apparent to the 

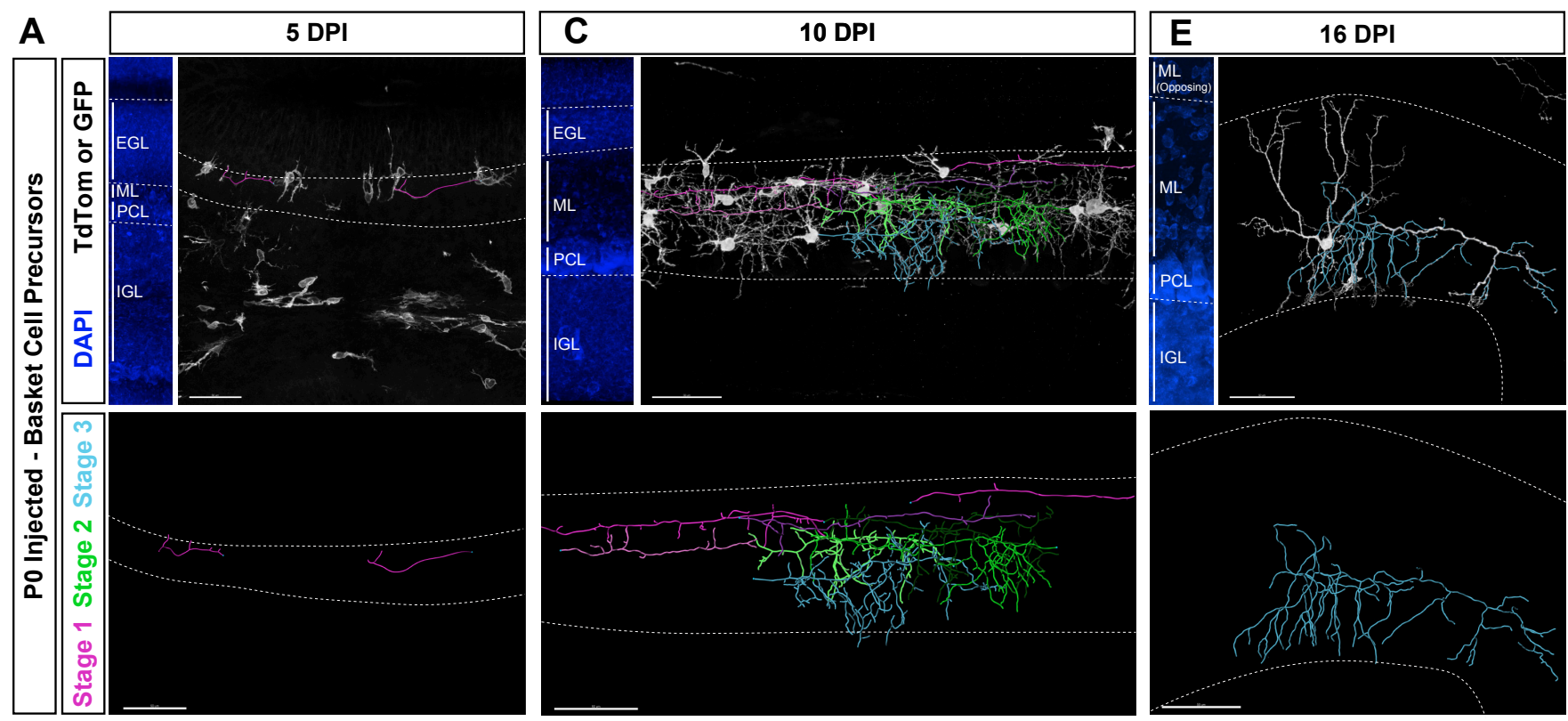

B

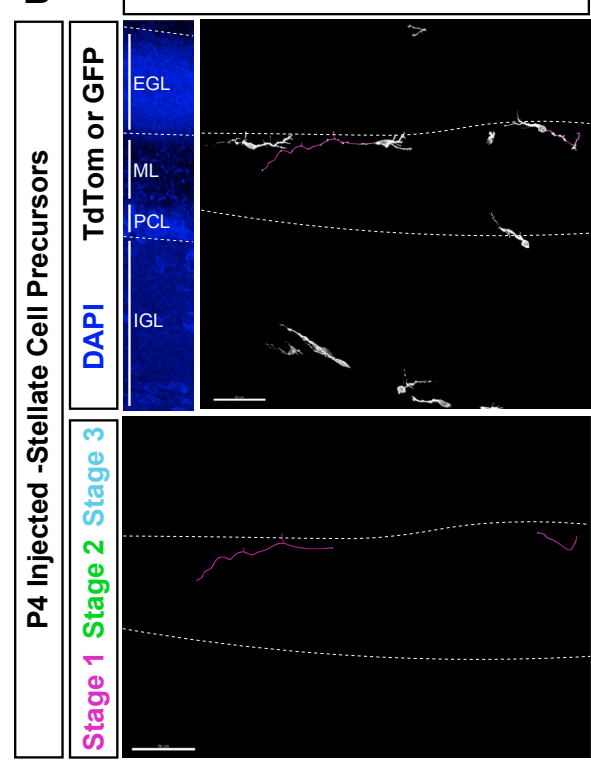

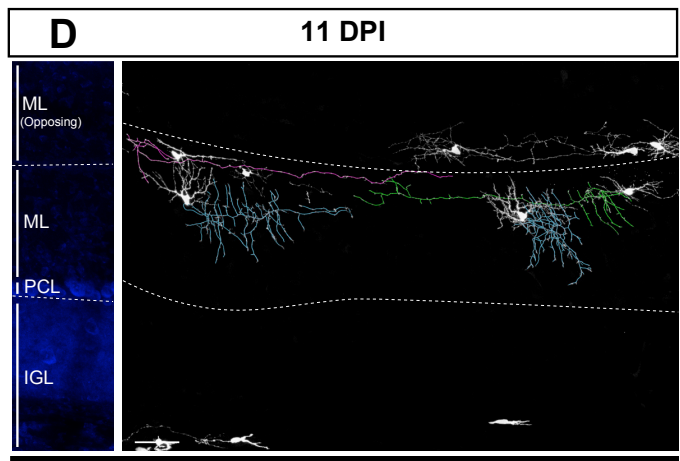
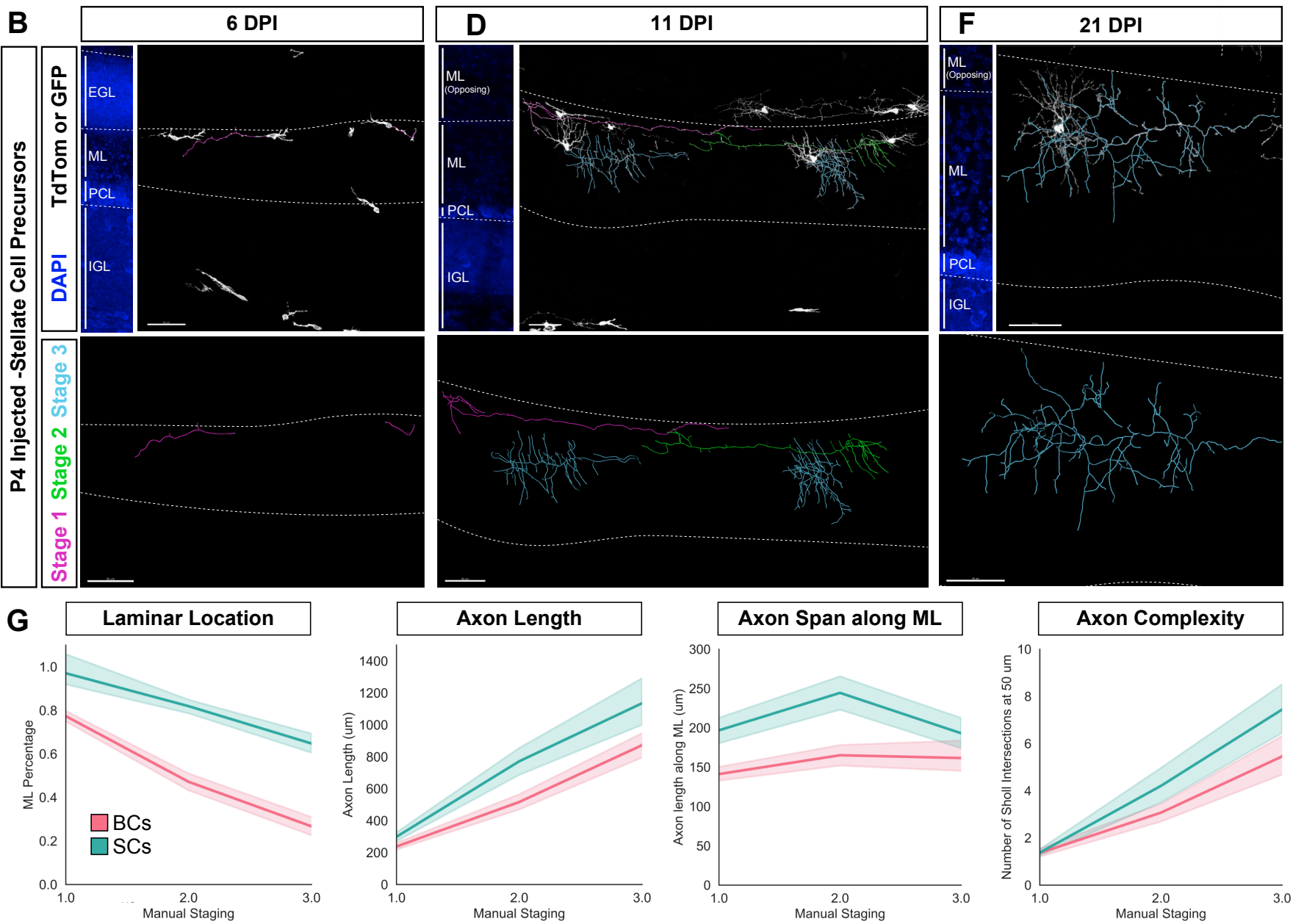

Figure 8. Comparisons of axonogenesis between BC- and SC-fated MLIs. (A-F) Fluorescently-labeled MLIs (top panels, greyscale) and axonal reconstructions (top and bottom panels). BCs were labeled by TMX injection at P0 (A,C,E) and SCs were labeled by P4/5 injection (B,D,F). Dashed lines demarcate the ML. Images of DAPI-stained cerebellar cortex are shown in the top left panels. (A) Migrating BCs at 5 days post-TMX injection (DPI) extend a trailing process, the presumptive axon (magenta). (B) Migrating SCs at $6 \mathrm{DPI}$ are in the superficial ML and extend a simple trailing process (magenta). (C) At 10 DPI, BCs display different stages of axonogenesis and are stratified in the $\mathrm{ML}$ according to axonal arbor progression. Immature BCs with a simple axon are located at the apical ML (pink or magenta traces, consistent with manual stage 1 of maturation). BCs with increasing 
axonal branching are located in the middle ML (green traces, Stage $2 \mathrm{MLIs}$; and blue traces, Stage 3). (D) SCs at $11 \mathrm{DPI}$ are similarly stratified in the ML according to progression of axonogenesis (Stage 1, magenta; Stage 2, green; Stage 3, blue). Note that the axonal spans of SCs at this stage are considerably larger than BCs at similar stages. (E) Representative BC axonal morphology nearing maturity (16 DPI, blue). (F) Representative SC axonal morphology nearing maturity (25 DPI, blue). All scale bars are $50 \mu \mathrm{m}$. (G) Line plots of single morphological parameters along stages of maturation, as per manual staging. BCs and SCs differ significantly at all stages of axon morphogenesis for ML laminar locations occupied, axon length, axon span along the $\mathrm{ML}$, and axon complexity.

329 trained eye at stage 1 and to a large extent at stage 2, as features common to maturing interneurons

330 visually outweigh minute subtype-specific differences. The inability to resolve the early segregation

331 by eye further emphasizes the power of large-scale, multi-dimensional analyses. Taken together,

332 visual inspections of MLI morphogenesis within the native ML environment confirmed pseudotime

333 outputs.

\section{A proof of concept for morphology-based predictive cell-typing of single neurons}

335 So far, we have shown that subtyping MLIs can be achieved at the population level using pseudotime

336 trajectory inference. We next asked whether our dataset and approach may be useful for subtyping

337 novel MLIs with single neuron resolution. We reasoned that by embedding single MLI traces into

338 the established PHATE-generated trajectory, one could infer characteristics such as maturity and

339 identity, using analogous properties of the nearest residing neighbor. As such, we propose the
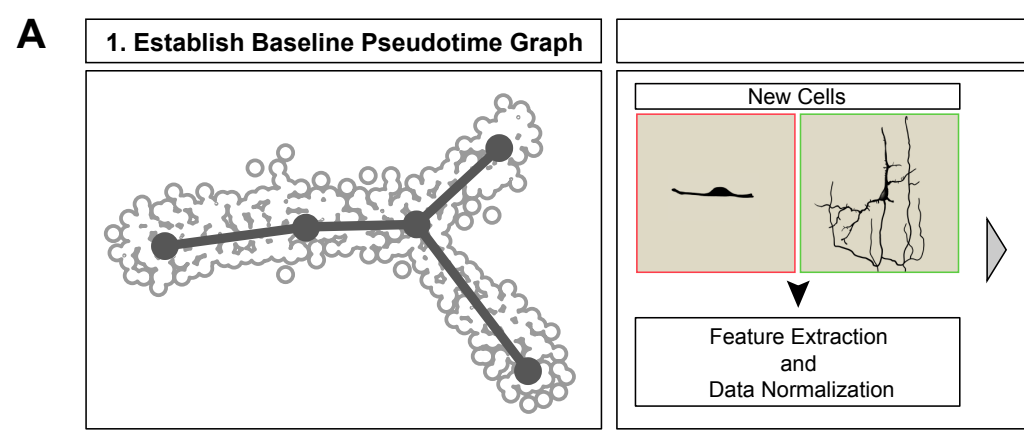

\section{Predictive Cell Typing}

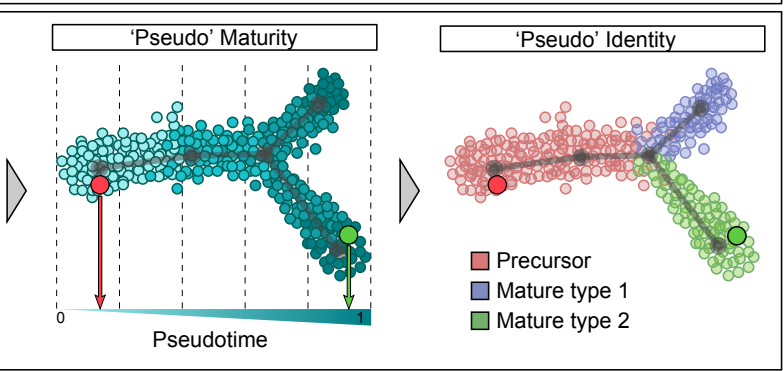

B Established pseudotime graph

C
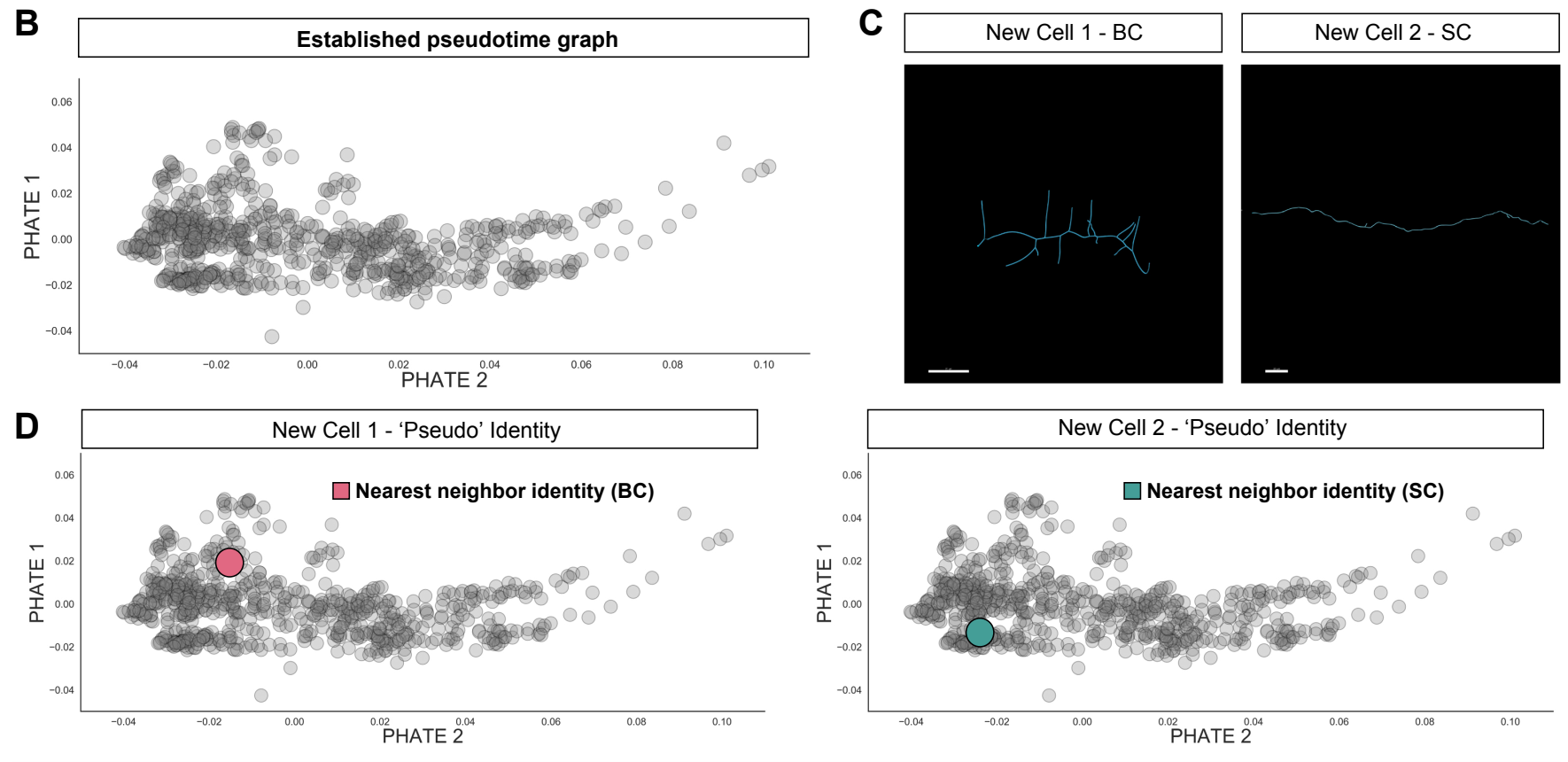

Wang and Lefebvre, 2020 | Diversification of cerebellar interneurons 
Figure 9. Proof of concept for predictive cell typing using morphological pseudotime. (A) Schematic for workflow of predictive cell typing using morphometric features. 1. Establish baseline pseudotime framework through large-scale single neuron profiling. 2. Obtain reconstructions for new cells, using previously established parameters. 3. Normalize new cell data to dataset. 4. Determine "pseudo-maturity" (left) pseudo-identity (right) of new cells. (B) Pseudotime trajectory established from Figure 7. (C) Reconstructions for two new cells that were not included in previous pseudotime analyses. Cell 1 was labelled at P0, and therefore is a presumptive BC, while cell 2 was labelled at P5, and therefore is a presumptive SC. (D) 'Pseudo' identities of new cell 1 (left) and new cell 2 (right). Color of highlight indicates the identity of the nearest neighbor (BC cell 1 and SC for cell 2 ). Scale bars are $20 \mu \mathrm{m}$.

340 terms 'pseudo-maturity' and 'pseudo-identity', as the two cells likely do not reside within identical 341 locations within the multi-dimensional data space, but one will nonetheless inform the identity of 342 the other (Figure 9A). As a proof of concept, we saved the coordinates of the PHATE pseudotime 343 manifold as a baseline operator (Figure 9B), and obtained new MLI reconstructions and their 344 morphometric signatures (Figure 9C). The novel cells were embedded into the PHATE manifold 345 as a new data point, followed by identification of the nearest neighbor (Figure 9D). We confirmed 346 that the 'pseudo-identity' for both of the cells analyzed matched that of their true terminal identity, 347 as determined by the corresponding time of TMX induction (Figure 9D). Given results from the 348 nearest neighbors analyses, we expect the accuracy of predicted 'pseudo-identities' to be within 349 the $75-90 \%$ range (Figure $7 G$ ). Taken together, we present a resource for current and future MLI 350 researchers for predictive morphological cell-typing, and offer a proof of concept for other neuronal 351 populations.

\section{Discussion}

353 Over 130 years ago, Santiago Ramón y Cajal took advantage of the accessible and compact 354 organization of the cerebellar MLIs to propose and substantiate the neuron doctrine (Cajal 1888; 355 Cajal 1911; Sotelo 2015). In homage to the original master, we re-examined the morphological diversity of MLIs using genetic and computational methods of the modern era. We show through clustering and dimensionality reduction approaches that MLIs divide into the basket and stellate cell subtypes, thus demonstrating that MLI heterogeneity is best described by the two populations rather than the continuous one population model. Iterative removal of morphological parameters identified the axonal arbor as the defining feature for MLI classification. Building on this finding, we compared axonogenesis of basket- and stellate-fated cells by combining genetic lineage tracing with a novel pseudotime trajectory inference approach. To validate this approach, MLI reconstructions were spatially and temporally annotated and visualized to confirm their developmental progression along pseudotime. In doing so, we determined that MLI subtype identities emerge during migration prior to reaching sites of final integration. Finally, we present our dataset as a tool for predictive subtyping of MLIs and offer a proof-of-concept for other neuronal populations.

Our study demonstrates the power of large-scale morphological quantifications to define neuronal subtypes and the anatomical and developmental features that distinguish them. As this approach can be widely applied to study morphological diversification, we highlight strengths and limitations. First, we identified a BC-SC division at maturity supported by multiple algorithms. Since 
371 we adhered to conservative clustering rules, it is possible that the heterogeneity among SCs may 372 parse into further subtypes with additional modalities. For instance, the early-born SCs in the lower

373 ML presented divergent migratory and morphological features during pseudotime compared to 374 the remaining SCs, suggesting that this population might represent another discrete subtype.

375 Second, genetic methods for targeted and high-resolution labelling of MLIs allowed for sampling 376 throughout their developmental progression. Large numbers of traces were required to adequately 377 capture all stages of MLI morphogenesis, which can slow throughput due to the need for sparse 378 labelling. Sampling can be improved by multicolor fluorescent reporters. Third, we provide the 379 first demonstration of pseudotime trajectory inference using neuronal morphological information. 380 As such, our analyses were dependent on pseudotime and modeling algorithms developed for 381 transcriptomic and proteomic datasets. While we obtained a reasonably robust trajectory for aligning 382 MLI identities, this approach might benefit from algorithms optimized for morphology. Fourth, 383 lineage tracing and pseudotime ordering of snapshots acquired across two weeks of development enabled the characterization of MLI morphogenesis. Although morphological pseudotime is an approximation of changes occuring in real time, it proved useful for delineating subtype-specific development and extracting statistically meaningful features. Altogether, the application of trajectory inference methods to quantitative morphological data is not only novel but also produced new insights into MLI diversification, which we detail below.

\section{A revised taxonomy of MLIs}

The long-standing debate of whether MLIs constitute one or multiple cell types reflects an enduring motivation to classify neurons into a systematic framework. The classical basket/stellate cell division is based on canonical patterns displayed by deep BCs and superficial SCs, which in our study only accounted for half of the total MLI population (Eccles et al. 1967; Palay and Chan-Palay 1974; Sotelo 2015). From observations of intermediate BC-SC morphologies, Cajal proposed that MLIs constitute a single cell type with continuously varying properties (Cajal et al. 1995; Sotelo 2015). In support of the 'one population' model, several studies demonstrated systematic variations of anatomical and electrophysiological features across molecular layer depth (Paula-Barbosa et al. 1983; Rakic 1972; Rieubland et al. 2014). Multivariate analysis of MLI anatomy similar to the one taken here failed to segregate the population by principal component analysis (Sultan and Bower 1998). This study was limited to twenty-six MLIs and contained few cells in the upper molecular layer due to the stochastic nature of Golgi labelling. With genetic access to MLIs that enhanced sampling across laminar positions, we obtained a clear BC-SC division among our dataset, which was confirmed through UMAP, t-SNE, hierarchical clustering, and statistical analyses.

Interestingly, the continuous heterogeneity we observed across the SC group reconciles both sides of the MLI taxonomy debate. Several SC morphometric quantifications, including dendritic and soma features, co-varied with laminar position in a manner that is consistent with the continuous 
407 variation previously attributed to the entire MLI population (Cajal et al. 1995; Rakic 1972; Rieubland 408 et al. 2014; Sultan and Bower 1998). Moreover, we did not observe bimodal distributions for any of 409 the morphological parameters that would indicate further divisions. Together, our findings support 410 a continuous variation model for describing the SC population. The spatial variation in SC identity 412 is not limited to anatomical properties but extends to functional ones. Variations in MLI connectivity 413 patterns across the molecular layer depth exert different influences on MLI network activity and 414 Purkinje cell firing (Arlt and Häusser 2020; Rieubland et al. 2014). Increasingly, single cell data 415 are revealing the prevalence of continuous variation in the organization of neuronal populations 416 (Cembrowski and Menon 2018; Cembrowski and Spruston 2019). For instance in the striatum and 417 hippocampus, graded RNA expression within discrete cell types also correlate with spatial positions 418 that reflect local or gross (i.e. dorsal-ventral axis) anatomical organization (Gokce et al. 2016; Arlt 419 and Häusser 2020; Muñoz-Manchado et al. 2018; Harris et al. 2018; Cembrowski et al. 2018; 420 Cembrowski et al. 2016). A few examples of neuronal subtypes that exhibit continuous variation in 421 molecular and electrophysiological signatures have been highlighted so far (Muñoz-Manchado et 422 al. 2018; Gouwens et al. 2019; Scala et al. 2020), but meaningful relationships between variations in 423 molecular, functional, and morphological properties remain unknown. Single cell characterizations 424 of transcriptional and functional properties of cerebellar neurons are underway (Zeisel et al. 2018; 425 Kozareva et al. 2020). A further understanding of MLI biology will come from investigations of 426 correspondence between modalities.

\section{MLI axonogenesis begins during migration}

428 In this study, we demonstrated the coupling of MLI axonogenesis with migration. Live imaging 429 studies of MLI migration have reported the extended, circular trajectory that includes four distinct 430 phases of radial and tangential migration through the molecular layer depth (Cameron et al. 2009; 431 Wefers et al. 2017; Wefers et al. 2018). These studies did not distinguish between basket and 432 stellate cells. Through pseudotime trajectory inference and confirmation in situ, we show that MLI 433 axon development is prolonged and temporally overlaps with multiple phases of migration. Both 434 young BCs and SCs in the apical ML exhibit elongated soma morphologies and extend a single 435 prospective axon. The timing, location, and migratory morphology of these MLIs are consistent 436 with the first tangential phase of ML migration (Cameron et al. 2009). These simple neurites are 437 consistent with descriptions of trailing processes that emerge during migration of some interneuron 438 populations (Lim et al. 2018; Marín et al. 2010; Sakakibara and Hatanaka 2015). In the cortical plate 439 for example, a subpopulation of radially migrating interneurons leave behind a trailing process that 440 remains stationary and eventually develops into the axonal arbor (Lim et al. 2018). Live imaging 441 studies of MLI migration did not report the presence of trailing processes nor axon formation 442 (Wefers et al. 2017; Cameron et al. 2009). Pseudotime modeling on the other hand, indicates that $443 \mathrm{MLI}$ axonal arborization initiates with a single trailing process that elongates along the apical ML 444 prior to the emergence of collaterals and branch remodeling. In a study which charted BC axon 
445 morphogenesis, secondary axon collaterals extended towards the PC somas through guidance 446 from extracellular guidance cues, as opposed to direct cell-cell interactions (Telley et al. 2016). 447 Although they did not account for the displacement of BCs during the process of axonogenesis, our 448 findings are consistent with the existence of such extracellular gradients for directing MLI migration 449 and axon guidance. Future studies on MLI migration should shed light on the identity and properties 450 of these molecules, which will likely resemble a gradient along the ML.

\section{Early emergence of MLI identities}

452 MLIs are the last category of interneurons generated in the cerebellum, as part of the Pax2453 expressing GABAergic interneuron lineage that originates from a single multipotent pool of 454 progenitors in the ventricular zone (Hoshino et al. 2005; Leto et al. 2006; Zhang and Goldman 1996; 455 Maricich and Herrup 1999; Weisheit et al. 2006). Proliferating MLI precursors exit the ventricular 456 zone and migrate to their secondary germinative region in the cerebellum, the prospective white 457 matter (PWM; Leto et al. 2006; Sotelo 2015). As such, MLls represent an interesting example in 458 the nervous system where proliferating progenitors migrate to an intermediate zone for further 459 expansion and terminal division. Since MLI fates are not intrinsically determined nor restricted 460 at birth, they are influenced by instructive cues encountered as they migrate from the PWM to 461 their final position in the molecular layer (Leto et al. 2009). Intriguingly, birth-dating studies have 462 demonstrated that post-mitotic MLIs remain in the PWM for 1-4 days following terminal division 463 (Leto et al. 2009). In Leto et al., comparisons between transplantation of dissociated neurons and 464 solid PWM grafts suggested that the PWM microenvironment may be a source of instructive cues 465 for MLI fate specification. The significance of this protracted and variable period of development 466 in the PWM on MLI differentiation remains unclear. Moreover, whether the inside-out placement of 467 MLIs and subtype-specific phenotypes within the mature ML is restricted by birthdate or the time 468 of PWM exit is unknown.

469 Through genetic lineage tracing and pseudotime trajectory inference, we determined that 470 MLI subtype identities emerge during early phases of migration in the ML, and that a subset of SCs 471 are generated along with BCs. The BC and SC lineages were separable across developmental 472 stages for a number of morphometric parameters, including total axon length, axon arbor span, and 473 laminar locations. This early divergence was further confirmed by the Palantir- and PHATE-generated 474 pseudotime trajectories, together revealing cluster-specific enrichments for BC- or SC-fated cells 475 along all developmental states. Although transplantation studies suggest that commitment to MLI 476 terminal identities remains plastic (Leto et al. 2009), our data indicate that MLI fates are instructed 477 earlier. Moreover, we demonstrated that MLI subtype identities are not dictated by ML laminations. 478 Lower ML SCs are among the early-born MLIs, occupy similar laminar locations as BCs, but were 479 separable from the BC lineage through pseudotime. Additionally, SCs are not restricted to upper $480 \mathrm{ML}$ positions but instead are distributed through the depth of the ML. Together, we propose that MLI 
481 laminar positioning is determined at the time of terminal division. However, subtype identities are 482 shaped by the timing of PWM exit or cues from the nascent ML. Although BCs and lower ML SCs

483 are born at similar times, lower ML SCs might adopt a different fate by taking longer to traverse 484 through the PWM and ML prior to axonogenesis. MLI lineage-specific profiling and manipulations 485 are required to further identify the source and timing of subtype specification.

\section{Concluding Remarks}

488 In conclusion, our study leveraged computational methods to characterize MLI development with 489 single-cell resolution and temporal coverage, while annotating individual cells using anatomical 490 and lineage information. Such approaches will inform future studies for cues that shape neuronal 491 patterning and for linking local variations in connectivity patterns to function. Importantly, restoring 492 anatomy and spatial-temporal contexts to single-cell profiling will provide the integrated descriptions 493 needed for understanding how diverse and complex neuronal subtypes arise and assemble into 494 circuits.

\section{Material and methods}

\section{Mouse strains}

Mouse lines used in this study have previously been described. GABAergic neuron targeting Gad2ires-Cre (JAX accession \#: 010802; Taniguchi et al. 2011) and MLI-specific Ascl1-CreERT2 (JAX \#: 012882; Kim et al. 2011) were obtained from Jackson Laboratories. Cre reporter lines Ai14 Rosa-CagLSL-TdTomato (JAX \#: 007908; Madisen et al. 2010) and RosamT/mG (JAX \#: 007576; Muzumdar et al. 2007) were obtained from Jackson Laboratories. Mice were maintained on a C57/B6J or mixed C57/B6J and FVB background.

All experiments were carried out in accordance with the Canadian Council on Animal Care guidelines for use of animal in research and laboratory animal care under protocols approved by the Centre for Phenogenomics Animal Care Committee (Toronto, Canada) and the Laboratory Animal Services Animal Care Committee at the Hospital for Sick Children (Toronto, Canada).

\section{Virus labelling of neurons}

Recombinant Brainbow AAV9-hEF1a-LoxP-TagBFP-LoxP-eYFP-LoxP-WPRE-hGH-InvBYF and AAV9hEF1a-LoxP-mCherry-LoxP-mTFP-LoxP-WPRE-hGH-InvCheTF viruses (Cai et al. 2013) were obtained from Pennsylvania Vector Core. $\sim 1 \times 10^{12}$ viral genome particles per $\mathrm{mL}$ of each Brainbow virus was prepared in sterile phosphate-buffered saline (PBS, $\mathrm{pH}=7.4$ ).

To introduce virus into the cerebellum, P0-14 pups (P0 for PCs, P5-14 for MLIs) were anaesthetized with either ice (P5 or younger) or isoflurane using a rodent anesthesia machine (>P6 and older, 4\% in O2). A $25 \mathrm{G}$ needle was used to make a small puncture into the caudal-medial position of the right cortical lobe, and $1.5 \mathrm{uL}$ of rAAV virus was injected into the lateral ventricles with a Hamilton syringe and 33G 
blunt-ended needle. Injection procedures were repeated for the left cortical lobe for bilateral labeling of neurons. Animals were sacrificed and cerebellums dissected at P75 for mature classifications.

\section{Tamoxifen induced labelling of neurons}

Tamoxifen (Sigma, T5648) was dissolved in corn oil (Sigma, C8267) to a concentration of $10 \mathrm{mg} / \mathrm{mL}$ (high dose injections; Figure 5C, middle and right panels), $0.5 \mathrm{mg} / \mathrm{mL}$ (low dose injections for sparse labeling using the $\mathrm{mTmG}$ Cre reporter), or $0.05 \mathrm{mg} / \mathrm{mL}$ (low dose injections for sparse labeling using Ai14-TdTomato Cre reporter). P0 - P7 pups were put on ice for 1 minute for anesthesia and to reduce oil leakage following injections. A $0.5 \mathrm{cc}$ insulin syringe (BD) was used to introduce $10-20 \mathrm{uL}$ of tamoxifen solution through intraperitoneal (I.P.) injections. The needle tip was held inside the pup for 20 seconds to prevent excessive leakage. Subcutaneous injections can be substituted for labeling of stellate cells (P4 - P7 injections), but I. P. injections are necessary for capturing basket cells in our postnatal injection scheme due to its faster acting nature for tamoxifen introduction and activation. Pups were sacrificed at least 3 days post-injection.

\section{Histology}

Mice were either anesthetized by hypothermia (P5 or younger) or under isofluorane (4\% in $\mathrm{O} 2$ for induction; $1.5-2 \%$ in $\mathrm{O} 2$ for maintenance), and transcardially perfused with physiological saline solution (0.9\%; Baxter) followed by $4 \%$ paraformaldehyde (PFA) in PBS. Animals were perfused by the gravity perfusion method. Brains were post-fixed in $4 \%$ PFA overnight at $4{ }^{\circ} \mathrm{C}$ or for 3.5 hours at room temperature (RT).

100 um sagittal sections of the vermis cerebellum were sectioned using a vibratome (Leica). Sections were incubated for 3.5 hours in blocking buffer $(0.5 \%$ Triton-X, $4 \%$ normal donkey serum in PBS), and incubated for 72 hours at $4^{\circ} \mathrm{C}$ with primary antibodies. Following $3 \times 15$ min PBST (0.5\% Triton-X) washes, sections were incubated for 3.5 hours at RT with Alexa-conjugated secondary antibodies (Invitrogen or Jackson ImmunoResearch). Sections were mounted onto glass microscope slides, coverslipped using Fluoromount G (Southern Biotech). Nuclei were labelled using DAPI or NeuroTrace Nissl 435/455 (Invitrogen).

Primary antibodies used for this study were as follows: chicken anti-GFP (1:2000, Aves Laboratories, GFP-1010); rabbit anti-mCherry (1:500, Kerafast, EMU106); rat anti-TFP (1:500, Kerafast, EMU104), guinea pig anti-TagBFP (1:500, Kerafast, EMU108); Rabbit anti-RFP (1:1000, Rockland, 600401-379); goat anti-Parvalbumin (1:1000, Swant, PVG213), and rabbit anti-Calbindin (1:1000, Sigma, C9848).

\section{Confocal imaging}

Images of single MLIs were taken on a Leica SP8 scanning confocal microscope, using a 40X oil objective (NA = 1.3). Z-stacks were collected with a 0.5 um (mature dataset) or 1 um (developmental dataset) step size throughout the depth of the cells, to encapsulate the entire dendritic and axonal arbor. Only neurons 
where arbors were not cut off by the vibratome were imaged and analyzed. The Z-compensation feature was used to avoid signal saturation throughout the depth of the tissue. Images were acquired with slightly different $X Y$ pixel sizes to accommodate varying arbor sizes, and to be able to capture single MLIs within one field of view. XY pixel sizes used were maintained at $\sim 135 \mathrm{~nm}$.

\section{MLI counts}

To account for the percent laminar distribution of singly labelled MLIs following Brainbow injections, we divided the ML into four strata, and counted the number of labelled MLIs within each strata. The XScope app was used to create a $1 \times 4 \mathrm{grid}$, which was resized to match the area of the ML. The number of MLI were then counted within each strata, using the cell counter plugin in FIJI (Schindelin et al. 2012). Each counting frame additionally consisted of one inclusion and one exclusion edge, and cells were counted if found entirely within the counting frame or overlapping with the inclusion edge but not the exclusion edge.

\section{Manual curation of MLI morphologies}

Canonical BCs and SCs were identified according to standards in literature (Amat et al. 2017; Sergaki et al. 2017; Gaffield and Christie 2017; Sultan and Bower 1998). Canonical BCs were identified by: 1) soma location within the bottom $1 / 3$ of the $\mathrm{ML}, 2$ ) a long horizontal axonal shaft which gives rise to basket terminals, and 3) a dendritic arbor which reaches the apical ML. Canonical SCs were identified by: 1) soma location within the upper $1 / 2$ of the $\mathrm{ML}, 2$ ) absence of basket terminals, 3 ) radially oriented dendritic and axonal arbors. The manually curated set of BCs likely contained fewer cells than both hierarchical clustering and UMAP analyses due to our strict laminar location cut-off.

\section{Morphological reconstructions}

$3 \mathrm{D}$ reconstructions for single neuron morphologies were completed in Imaris (Bitplane). Dendritic and axonal arbors were semi-automatically reconstructed using the filament tracer (autodepth mode). Surface render of the cell soma was performed using the surfaces module. We note that due to the membrane targeted nature of the Brainbow fluorophores, the soma volume data for mature MLIs more accurately describes the volume of the cell membrane, and not the entire somata.

\section{Data compilation of single neuron anatomical features}

For each neuron, we: 1) registered the spatial coordinates to note the cell location and folia of origin in the vermis cerebellum; 2) performed surface rendering for the cell soma, 3) performed morphological reconstructions, and 4) extracted 27 quantitative features to describe the dendrites, axon, somata, and location (Table 1).

Basic morphological features were exported from Imaris using the statistics tab. These include: axon/dendrite length, number of Sholl intersections at 10, 50, 100, 150, and $200 \mathrm{um}$, mean/max branch level, axon straightness, and volume of somatic render. The remaining features were manually compiled: 
Mature dataset:

1. The number of filopodia was calculated as the number of terminal branches under $1.5 \mathrm{um}$ in length.

2. Filopodia density was calculated by normalizing total filopodia numbers to the dendrite length.

3. Z-depth, height of ML covered by dendrites/axons, and axonal span along the ML were measured using the measurement tool in Imaris.

4. Radial symmetry of a cell was manually graded between 1-4, but was not included in the final clustering dataset due to the qualitative nature of the assessments.

5. For analyses of upwards- and downwards-oriented axon collaterals (numbers, length, and percentage), collaterals directed towards the apical or basal ML were highlighted in Imaris, and the corresponding data were compiled from the statistics tab. For collaterals which were accurately angled, only branches more than 30 degrees away from the main axon were included in the analysis.

6. Presence of axon-carrying dendrites were noted for cells where the axon initial segment originates from the basal dendrites, not the soma.

7. Folial location was noted in folia $1,3,5,6,7,8,9$, and 10 . This data was not included in the final clustering analyses, but used for confirmation that MLI identities are not biased by the cell's folia of origin.

8. Relative molecular layer (ML) position was measured using the measurement tool within slice mode. ML heights were calculated as the distance from the top of the PCL to the top of the ML. MLI soma heights were calculated as the distance from the top of the PCL to the centre of the MLI soma. MLI laminar locations were calculated as MLI soma height / ML height.

9. For each cell, the number of full baskets (one which fully envelopes the corresponding PC soma) with pinceau formations were counted in addition to the number of full baskets without pinceau formations, and the number of half baskets (one which less than entirely envelops the PC soma). Number of baskets were then calculated using a weighted system, according to the proxied functional significance of each basket. In this way, full baskets with pinceau formations were given a weight of 1 , full baskets without pinceau formations were given a weight of 0.75 , and half baskets were given a weight of 0.5 .

10. The number of primary dendrites were counted as the number of branches which arises from the soma.

\section{Developmental dataset:}

1. Overlapping parameters were compiled following similar protocols as the mature dataset. These include axonal height, depth and span information, measurements of upwards- and downwards-oriented axon collaterals, laminar locations, and the number of primary dendrites.

2. The number of axonal branches which reached into the PCL were counted, to account for developmental changes as most basket-forming cells have not formed full basket formations at the timepoints analyzed. 3. Soma diameter for each cell was measured along three dimensions using the measurement tool within slice mode $(X=$ direction along the $P C L, Y=$ direction perpendicular to the $P C L$, and $Z=$ direction along parallel fibers). The final somatic volume was approximated using the ellipsoid volume equation: $\mathrm{V}=4 / 3 \pi \times \mathrm{X}$-diameter $\times \mathrm{Y}$-diameter $\times \mathrm{Z}$-diameter. 
We note that although a full 3D volumetric render of the soma similar to what was done for the mature dataset would have been more accurate, the process was time and computationally intensive. Approximating the soma volume allowed for the large-scale nature of our study.

\section{Clustering analyses}

Prior to morphometric clustering, each dataset was zero mean standardized as previously described (Lanjakornsiripan et al. 2018). t-SNE was performed with the Rtsne package (version 0.15) for the $R$ statistical environment (R Foundation for Statistical Computing 2018; version 3.5.1) with the following parameters: seed $=510$, theta $=0$, max_iter $=10,000$, and perplexity $=7$. The elbow method and silhouette analysis were performed using the NBClust package (version 3.0) in R. UMAP analysis was performed in Python (Python Software Foundation n.d.; version 3.7.3) using the umap and sklearn packages, with the following parameters: min_neighbors $=7$, min_dist $=0.1$, metric = 'euclidean'. Hierarchical clustering was performed using the SciPy.cluster.hierarchy package in Python, using the Ward's method (Ward 1963). SC subclades were identified visually through curation of the corresponding MLI traces.

\section{Camera Lucida illustrations}

Camera Lucida traces of dendritic morphologies were created using the Affinity Designer (Serif) app on an iPad (Apple). Max projection of the dendritic arbors were obtained in FIJI. The resulting image was inserted in Affinity Designer. Traces of the dendritic and somatic outlines were completed using an Apple pencil.

\section{Expert directed pseudotime based on dendritic maturity}

Manual staging of MLI morphometric maturation was performed based on our 4 stage maturation scheme (Figure 6 - Figure Supplement 1). Additionally, migratory precursor cells in Figure 6M were identified as neurons which had a migratory leading process (similar to those found on stage 1 cells), but which did not possess an observable trailing process.

\section{Pseudotime trajectory inference using Palantir}

We adapted the Palantir pseudotime algorithm to align our single cell snapshots in the developmental space (Setty et al. 2019): 1) The developmental dataset was zero mean standardized outside of Palantir. This step was performed individually for the total MLI dataset, and the early-born MLI subset; 2) the normalized datasets were imported into Palantir as a normalized dataframe; 3) principal component analysis was performed to determine the principal components; 4) Diffusion maps of the data were obtained to determine the low dimensional phenotypic manifold of the data, with n_components $=5 ; 5$ ) $\mathrm{t}-\mathrm{SNE}$ representation of the data was created in the embedded space; 6) MAGIC imputation (van Dijk et al. 2018) was used to map trends for each morphological parameter onto the t-SNE map; 7) starter cells were manually assigned following expert curation of the corresponding morphology traces; 8 ) clustering 
was performed using the Phenograph package and the Louvain method (Levine et al. 2015), with $\mathrm{k}=20$.

\section{Trajectory inference using PHATE}

We adapted the PHATE algorithm for aligning our single cell snapshots in the developmental space (Moon et al. 2019): 1) The developmental dataset was zero mean standardized in the same way as our Palantir analyses; 2) the normalized datasets were imported into PHATE as a normalized dataframe; 3 ) the dataframe was used to generate a PHATE estimator object with the following parameters: $k n n=3 ; 4$ ) diffusion pseudotime was performed on the PHATE operator using Scanpy (Wolf et al. 2018); 5) expert directed maturity stages were projected onto the PHATE manifold as a color label.

\section{Nearest neighbor analyses}

The nearest neighbor analysis were performed following PHATE-based pseudotime trajectory inference by: 1 ) binning the pseudo-timeline into 0.2 unit increments; 2 ) identifying the nearest neighbor for each MLI reconstruction within the PHATE manifold; 3) determining whether the terminal identity of the nearest neighbor is the same or different from the cell in question; 4) determining the percentage of cells within each pseudotime bin with a neighbors of the same terminal fate.

\section{Experimental design and statistical analysis}

Morphological reconstructions for the 79 mature MLIs were compiled from 9 P75 mice across cerebellar folias. Developmental axonal reconstructions for the $732 \mathrm{MLIs}$ were compiled from 32 mouse pups, which included the following injection to collection timelines: 1) P0 to P5; 2) P0 to P7; 3) P0 to P8; 4) P0 to P10; 5) $\mathrm{P} 0$ to $\mathrm{P} 13$; 6) $\mathrm{P} 0$ to $\mathrm{P} 16$; 7) $\mathrm{P} 0$ to $\mathrm{P} 25 ; 8) \mathrm{P} 1$ to $\mathrm{P} 13$; 9) $\mathrm{P} 1$ to $\mathrm{P} 16$; 10) $\mathrm{P} 1$ to $\mathrm{P} 25$; 11) $\mathrm{P} 2$ to $\mathrm{P} 13$; 12$) \mathrm{P} 2$ to $\mathrm{P} 16$; 13) $\mathrm{P} 2$ to $\mathrm{P} 27$; 14) $\mathrm{P} 3$ to $\mathrm{P} 13$; 15) $\mathrm{P} 3$ to $\mathrm{P} 16$; 16) $\mathrm{P} 4$ to $\mathrm{P} 10 ; 17) \mathrm{P} 4$ to $\mathrm{P} 13$; 18) $\mathrm{P} 4$ to $\mathrm{P} 15 ; 19) \mathrm{P} 5$ to $\mathrm{P} 13$; 20) P5 to $\mathrm{P} 14 ; 21) \mathrm{P} 5$ to $\mathrm{P} 16$; and 22) P7 to P14. Animals of either sex were analyzed. The early born population for pseudotime analyses contained all PO injected samples, as well as a small number of mature P1 labelled cells which were confirmed as BCs due to the elaboration of soma-targeting axons. The late-born population contained all P4-P7 injected samples. Statistical analyses were performed using the GraphPad Prism software or the SciPy package in Python. Means of two groups were compared using the two-tailed student's $t$ test with the Mann-Whitney nonparametric test. Graphs were created using the Seaborn or Matplotlib packages in Python, or using GraphPad Prism.

\section{Acknowledgements}

This work was supported by a Canada Research Chair (Tier 2), a Sloan Fellowship in Neuroscience, an NSERC Discovery grant, a CIHR Project grant, and funding from the Hospital for Sick Children (to J.L.L.). We thank Dr. Shreejoy Tripathy and members of the Lefebvre lab for helpful comments on this manuscript, and Dr. Manu Setty for discussions on morphological pseudotime. We thank Scott Gigante for computational assistance for binning of pseudo-timelines. 


\section{Author contributions}

W.X.W and J.L.L. conceptualized and designed the project. W.X.W. performed experiments and computational analyses. W.X.W. and J.L.L. wrote the manuscript.

\section{References}

1. Altman, J., and Bayer, S.A. (1978). Prenatal development of the cerebellar system in the rat. I. Cytogenesis and histogenesis of the deep nuclei and the cortex of the cerebellum. J. Comp. Neurol. 179, 23-48.

2. Altman, J., and Bayer, S.A. (1997). Development of the cerebellar system: in relation to its evolution, structure, and functions (CRC Press).

3. Amat, S.B., Rowan, M.J.M., Gaffield, M.A., Bonnan, A., Kikuchi, C., Taniguchi, H., and Christie, J.M. (2017). Using c-kit to genetically target cerebellar molecular layer interneurons in adult mice. PLoS ONE 12, e0179347.

4. Arlt, C., and Häusser, M. (2020). Microcircuit rules governing impact of single interneurons on purkinje cell output in vivo. Cell Rep. 30, 3020-3035.e3.

5. Bae, J.A., Mu, S., Kim, J.S., Turner, N.L., Tartavull, I., Kemnitz, N., Jordan, C.S., Norton, A.D., Silversmith, W.M., Prentki, R., et al. (2018). Digital Museum of Retinal Ganglion Cells with Dense Anatomy and Physiology. Cell 173, 1293-1306.e19.

6. Becht, E., Mclnnes, L., Healy, J., Dutertre, C.-A., Kwok, I.W.H., Ng, L.G., Ginhoux, F., and Newell, E.W. (2018). Dimensionality reduction for visualizing single-cell data using UMAP. Nat. Biotechnol.

7. Brown, A.M., Arancillo, M., Lin, T., Catt, D.R., Zhou, J., Lackey, E.P., Stay, T.L., Zuo, Z., White, J.J., and Sillitoe, R.V. (2019). Molecular layer interneurons shape the spike activity of cerebellar Purkinje cells. Sci. Rep. 9, 1742.

8. Buttermore, E.D., Piochon, C., Wallace, M.L., Philpot, B.D., Hansel, C., and Bhat, M.A. (2012). Pinceau organization in the cerebellum requires distinct functions of neurofascin in Purkinje and basket neurons during postnatal development. J. Neurosci. 32, 4724-4742.

9. Cai, D., Cohen, K.B., Luo, T., Lichtman, J.W., and Sanes, J.R. (2013). Improved tools for the Brainbow toolbox. Nat. Methods 10, 540-547.

10. Cajal, S.R. (1888). Estructura de los centros neviosos de las aves.

11. Cajal, S.R. (1911). Histologie du système nerveux de l'homme \& des vertébrés.

12. Cajal, S.R. y, Swanson, N., and Swanson, L. (1995). Histology of the Nervous System of Man and Vertebrates (History of Neuroscience, No 6) (2 Volume Set) (Oxford University Press).

13. Cameron, D.B., Kasai, K., Jiang, Y., Hu, T., Saeki, Y., and Komuro, H. (2009). Four distinct phases of basket/stellate cell migration after entering their final destination (the molecular layer) in the developing cerebellum. Dev. Biol. 332, 309-324.

14. Cembrowski, M.S., and Menon, V. (2018). Continuous Variation within Cell Types of the Nervous System. Trends Neurosci. 41, 337-348.

15. Cembrowski, M.S., and Spruston, N. (2019). Heterogeneity within classical cell types is the rule: lessons from hippocampal pyramidal neurons. Nat. Rev. Neurosci. 20, 193-204.

16. Cembrowski, M.S., Bachman, J.L., Wang, L., Sugino, K., Shields, B.C., and Spruston, N. (2016). Spatial Gene-Expression Gradients Underlie Prominent Heterogeneity of CA1 Pyramidal Neurons. Neuron 89, 351-368.

17. Cembrowski, M.S., Wang, L., Lemire, A.L., Copeland, M., DiLisio, S.F., Clements, J., and Spruston, N. (2018). The subiculum is a patchwork of discrete subregions. Elife 7.

18. Clark, B.S., Stein-O'Brien, G.L., Shiau, F., Cannon, G.H., Davis-Marcisak, E., Sherman, T., Santiago, C.P., Hoang, T.V., Rajaii, F., James-Esposito, R.E., et al. (2019). Single-Cell RNA-Seq Analysis of Retinal Development Identifies NFI Factors as Regulating Mitotic Exit and Late-Born Cell Specification. Neuron 102, 1111-1126.e5.

19. Coifman, R.R., Lafon, S., Lee, A.B., Maggioni, M., Nadler, B., Warner, F., and Zucker, S.W. (2005). 
Geometric diffusions as a tool for harmonic analysis and structure definition of data: diffusion maps. Proc Natl Acad Sci USA 102, 7426-7431.

20. Costa, M., Manton, J.D., Ostrovsky, A.D., Prohaska, S., and Jefferis, G.S.X.E. (2016). NBLAST: rapid, sensitive comparison of neuronal structure and construction of neuron family databases. Neuron 91 , 293-311.

21. van Dijk, D., Sharma, R., Nainys, J., Yim, K., Kathail, P., Carr, A.J., Burdziak, C., Moon, K.R., Chaffer, C.L., Pattabiraman, D., et al. (2018). Recovering Gene Interactions from Single-Cell Data Using Data Diffusion. Cell 174, 716-729.e27.

22. Eccles, J.C., Sasaki, K., and Strata, P. (1967). A comparison of the inhibitory actions of Golgi cells and of basket cells. Exp. Brain Res. 3, 81-94.

23. Economo, M.N., Clack, N.G., Lavis, L.D., Gerfen, C.R., Svoboda, K., Myers, E.W., and Chandrashekar, J. (2016). A platform for brain-wide imaging and reconstruction of individual neurons. Elife 5, e10566.

24. Frechter, S., Bates, A.S., Tootoonian, S., Dolan, M.-J., Manton, J., Jamasb, A.R., Kohl, J., Bock, D., and Jefferis, G. (2019). Functional and anatomical specificity in a higher olfactory centre. Elife 8.

25. Gaffield, M.A., and Christie, J.M. (2017). Movement rate is encoded and influenced by widespread, coherent activity of cerebellar molecular layer interneurons. J. Neurosci. 37, 4751-4765.

26. Geirsdottir, L., David, E., Keren-Shaul, H., Weiner, A., Bohlen, S.C., Neuber, J., Balic, A., Giladi, A., Sheban, F., Dutertre, C.-A., et al. (2019). Cross-Species Single-Cell Analysis Reveals Divergence of the Primate Microglia Program. Cell 179, 1609-1622.e16.

27. Gokce, O., Stanley, G.M., Treutlein, B., Neff, N.F., Camp, J.G., Malenka, R.C., Rothwell, P.E., Fuccillo, M.V., Südhof, T.C., and Quake, S.R. (2016). Cellular Taxonomy of the Mouse Striatum as Revealed by Single-Cell RNA-Seq. Cell Rep. 16, 1126-1137.

28. Gouwens, N.W., Sorensen, S.A., Berg, J., Lee, C., Jarsky, T., Ting, J., Sunkin, S.M., Feng, D., Anastassiou, C.A., Barkan, E., et al. (2019). Classification of electrophysiological and morphological neuron types in the mouse visual cortex. Nat. Neurosci. 22, 1182-1195.

29. Grimaldi, P., Parras, C., Guillemot, F., Rossi, F., and Wassef, M. (2009). Origins and control of the differentiation of inhibitory interneurons and glia in the cerebellum. Dev. Biol. 328, 422-433.

30. Harris, J.A., Hirokawa, K.E., Sorensen, S.A., Gu, H., Mills, M., Ng, L.L., Bohn, P., Mortrud, M., Ouellette, B., Kidney, J., et al. (2014). Anatomical characterization of Cre driver mice for neural circuit mapping and manipulation. Front. Neural Circuits 8, 76.

31. Harris, K.D., Hochgerner, H., Skene, N.G., Magno, L., Katona, L., Bengtsson Gonzales, C., Somogyi, P., Kessaris, N., Linnarsson, S., and Hjerling-Leffler, J. (2018). Classes and continua of hippocampal CA1 inhibitory neurons revealed by single-cell transcriptomics. PLoS Biol. 16, e2006387.

32. Helmstaedter, M., Briggman, K.L., Turaga, S.C., Jain, V., Seung, H.S., and Denk, W. (2013). Connectomic reconstruction of the inner plexiform layer in the mouse retina. Nature 500, 168-174.

33. He, M., Tucciarone, J., Lee, S., Nigro, M.J., Kim, Y., Levine, J.M., Kelly, S.M., Krugikov, I., Wu, P., Chen, Y., et al. (2016). Strategies and tools for combinatorial targeting of gabaergic neurons in mouse cerebral cortex. Neuron 91, 1228-1243.

34. Hoshino, M., Nakamura, S., Mori, K., Kawauchi, T., Terao, M., Nishimura, Y.V., Fukuda, A., Fuse, T., Matsuo, N., Sone, M., et al. (2005). Ptf1a, a bHLH transcriptional gene, defines GABAergic neuronal fates in cerebellum. Neuron 47, 201-213.

35. Huang, Z.J., and Paul, A. (2019). The diversity of GABAergic neurons and neural communication elements. Nat. Rev. Neurosci. 20, 563-572.

36. Jiang, X., Shen, S., Cadwell, C.R., Berens, P., Sinz, F., Ecker, A.S., Patel, S., and Tolias, A.S. (2015). Principles of connectivity among morphologically defined cell types in adult neocortex. Science 350 , aac9462.

37. Kanari, L., Ramaswamy, S., Shi, Y., Morand, S., Meystre, J., Perin, R., Abdellah, M., Wang, Y., Hess, K., and Markram, H. (2019). Objective morphological classification of neocortical pyramidal cells. Cereb. Cortex 29, 1719-1735.

38. Kepecs, A., and Fishell, G. (2014). Interneuron cell types are fit to function. Nature 505, 318-326. 
39. Kim, E.J., Ables, J.L., Dickel, L.K., Eisch, A.J., and Johnson, J.E. (2011). Ascl1 (Mash1) defines cells with long-term neurogenic potential in subgranular and subventricular zones in adult mouse brain. PLoS ONE 6, e18472.

40. Kozareva, V., Martin, C., Osorno, T., Rudolph, S., Guo, C., Vanderburg, C., Nadaf, N.M., Regev, A., Regehr, W., and Macosko, E. (2020). A transcriptomic atlas of the mouse cerebellum reveals regional specializations and novel cell types. BioRxiv.

41. Lanjakornsiripan, D., Pior, B.-J., Kawaguchi, D., Furutachi, S., Tahara, T., Katsuyama, Y., Suzuki, Y., Fukazawa, Y., and Gotoh, Y. (2018). Layer-specific morphological and molecular differences in neocortical astrocytes and their dependence on neuronal layers. Nat. Commun. 9, 1623.

42. Lefebvre, J.L., Sanes, J.R., and Kay, J.N. (2015). Development of dendritic form and function. Annu. Rev. Cell Dev. Biol. 31, 741-777.

43. Leto, K., Carletti, B., Williams, I.M., Magrassi, L., and Rossi, F. (2006). Different types of cerebellar GABAergic interneurons originate from a common pool of multipotent progenitor cells. J. Neurosci. 26, 11682-11694.

44. Leto, K., Bartolini, A., Yanagawa, Y., Obata, K., Magrassi, L., Schilling, K., and Rossi, F. (2009). Laminar fate and phenotype specification of cerebellar GABAergic interneurons. J. Neurosci. 29, 7079-7091.

45. Leto, K., Bartolini, A., Di Gregorio, A., Imperiale, D., De Luca, A., Parmigiani, E., Filipkowski, R.K., Kaczmarek, L., and Rossi, F. (2011). Modulation of cell-cycle dynamics is required to regulate the number of cerebellar GABAergic interneurons and their rhythm of maturation. Development 138, 3463-3472.

46. Leto, K., Rolando, C., and Rossi, F. (2012). The genesis of cerebellar GABAergic neurons: fate potential and specification mechanisms. Front. Neuroanat. 6, 6.

47. Levine, J.H., Simonds, E.F., Bendall, S.C., Davis, K.L., Amir, E.D., Tadmor, M.D., Litvin, O., Fienberg, H.G., Jager, A., Zunder, E.R., et al. (2015). Data-Driven Phenotypic Dissection of AML Reveals Progenitor-like Cells that Correlate with Prognosis. Cell 162, 184-197.

48. Lim, L., Pakan, J.M.P., Selten, M.M., Marques-Smith, A., Llorca, A., Bae, S.E., Rochefort, N.L., and Marín, O. (2018). Optimization of interneuron function by direct coupling of cell migration and axonal targeting. Nat. Neurosci. 21, 920-931.

49. Madisen, L., Zwingman, T.A., Sunkin, S.M., Oh, S.W., Zariwala, H.A., Gu, H., Ng, L.L., Palmiter, R.D., Hawrylycz, M.J., Jones, A.R., et al. (2010). A robust and high-throughput Cre reporting and characterization system for the whole mouse brain. Nat. Neurosci. 13, 133-140.

50. Maricich, S.M., and Herrup, K. (1999). Pax-2 expression defines a subset of GABAergic interneurons and their precursors in the developing murine cerebellum. J. Neurobiol. 41, 281-294.

51. Marín, O., Valiente, M., Ge, X., and Tsai, L.-H. (2010). Guiding neuronal cell migrations. Cold Spring Harb. Perspect. Biol. 2, a001834.

52. Markram, H., Toledo-Rodriguez, M., Wang, Y., Gupta, A., Silberberg, G., and Wu, C. (2004). Interneurons of the neocortical inhibitory system. Nat. Rev. Neurosci. 5, 793-807.

53. Markram, H., Muller, E., Ramaswamy, S., Reimann, M.W., Abdellah, M., Sanchez, C.A., Ailamaki, A., Alonso-Nanclares, L., Antille, N., Arsever, S., et al. (2015). Reconstruction and simulation of neocortical microcircuitry. Cell 163, 456-492.

54. Mayer, C., Hafemeister, C., Bandler, R.C., Machold, R., Batista Brito, R., Jaglin, X., Allaway, K., Butler, A., Fishell, G., and Satija, R. (2018). Developmental diversification of cortical inhibitory interneurons. Nature 555, 457-462.

55. Mclnnes, L., Healy, J., and Melville, J. (2018). UMAP: Uniform Manifold Approximation and Projection for Dimension Reduction. ArXiv.

56. Mi, D., Li, Z., Lim, L., Li, M., Moissidis, M., Yang, Y., Gao, T., Hu, T.X., Pratt, T., Price, D.J., et al. (2018). Early emergence of cortical interneuron diversity in the mouse embryo. Science 360, 81-85.

57. Moon, K.R., van Dijk, D., Wang, Z., Gigante, S., Burkhardt, D.B., Chen, W.S., Yim, K., Elzen, A. van den, Hirn, M.J., Coifman, R.R., et al. (2019). Visualizing structure and transitions in high-dimensional 
biological data. Nat. Biotechnol. 37, 1482-1492.

58. Muñoz-Manchado, A.B., Bengtsson Gonzales, C., Zeisel, A., Munguba, H., Bekkouche, B., Skene, N.G., Lönnerberg, P., Ryge, J., Harris, K.D., Linnarsson, S., et al. (2018). Diversity of Interneurons in the Dorsal Striatum Revealed by Single-Cell RNA Sequencing and PatchSeq. Cell Rep. 24, 21792190.e7.

59. Muzumdar, M.D., Tasic, B., Miyamichi, K., Li, L., and Luo, L. (2007). A global double-fluorescent Cre reporter mouse. Genesis 45, 593-605.

60. Palay, S.L., and Chan-Palay, V. (1974). Cerebellar Cortex (Berlin, Heidelberg: Springer Berlin Heidelberg).

61. Paula-Barbosa, M.M., Tavares, M.A., Ruela, C., and Barroca, H. (1983). The distribution of stellate cell descending axons in the rat cerebellum: a Golgi and a combined Golgi-electron microscopical study. J. Anat. 137 ( Pt 4), 757-764.

62. Python Software Foundation Python Language Reference.

63. Rakic, P. (1972). Extrinsic cytological determinants of basket and stellate cell dendritic pattern in the cerebellar molecular layer. J. Comp. Neurol. 146, 335-354.

64. Rieubland, S., Roth, A., and Häusser, M. (2014). Structured connectivity in cerebellar inhibitory networks. Neuron 81, 913-929.

65. Rousseeuw, P.J. (1987). Silhouettes: A graphical aid to the interpretation and validation of cluster analysis. Journal of Computational and Applied Mathematics 20, 53-65.

66. R Foundation for Statistical Computing, R.C.T. (2018). R: A language and environment for statistical computing.

67. Saelens, W., Cannoodt, R., Todorov, H., and Saeys, Y. (2019). A comparison of single-cell trajectory inference methods. Nat. Biotechnol. 37, 547-554.

68. Sakakibara, A., and Hatanaka, Y. (2015). Neuronal polarization in the developing cerebral cortex. Front. Neurosci. 9, 116.

69. Scala, F., Kobak, D., Bernabucci, M., Bernaerts, Y., Cadwell, C.R., Castro, J.R., Hartmanis, L., Jiang, X., Laturnus, S.R., Miranda, E., et al. (2020). Phenotypic variation within and across transcriptomic cell types in mouse motor cortex. BioRxiv.

70. Scheibel, M.E., and Scheibel, A.B. (1954). Observations on the intracortical relations of the climbing fibers of the cerebellum; a Golgi study. J. Comp. Neurol. 101, 733-763.

71. Schilling, K., and Oberdick, J. (2009). The treasury of the commons: making use of public gene expression resources to better characterize the molecular diversity of inhibitory interneurons in the cerebellar cortex. Cerebellum 8, 477-489.

72. Schindelin, J., Arganda-Carreras, I., Frise, E., Kaynig, V., Longair, M., Pietzsch, T., Preibisch, S., Rueden, C., Saalfeld, S., Schmid, B., et al. (2012). Fiji: an open-source platform for biological-image analysis. Nat. Methods 9, 676-682.

73. Sergaki, M.C., López-Ramos, J.C., Stagkourakis, S., Gruart, A., Broberger, C., Delgado-García, J.M., and Ibáñez, C.F. (2017). Compromised survival of cerebellar molecular layer interneurons lacking GDNF receptors gfra1 or RET impairs normal cerebellar motor learning. Cell Rep. 19, 1977-1986.

74. Serra, D., Mayr, U., Boni, A., Lukonin, I., Rempfler, M., Challet Meylan, L., Stadler, M.B., Strnad, P., Papasaikas, P., Vischi, D., et al. (2019). Self-organization and symmetry breaking in intestinal organoid development. Nature 569, 66-72.

75. Setty, M., Kiseliovas, V., Levine, J., Gayoso, A., Mazutis, L., and Pe'er, D. (2019). Characterization of cell fate probabilities in single-cell data with Palantir. Nat. Biotechnol. 37, 451-460.

76. Sotelo, C. (2008). Viewing the cerebellum through the eyes of Ramón Y Cajal. Cerebellum 7, 517-522.

77. Sotelo, C. (2015). Molecular layer interneurons of the cerebellum: developmental and morphological aspects. Cerebellum 14, 534-556.

78. Sudarov, A., Turnbull, R.K., Kim, E.J., Lebel-Potter, M., Guillemot, F., and Joyner, A.L. (2011). Ascl1 genetics reveals insights into cerebellum local circuit assembly. J. Neurosci. 31, 11055-11069.

79. Sultan, F., and Bower, J.M. (1998). Quantitative Golgi study of the rat cerebellar molecular layer 
interneurons using principal component analysis. J. Comp. Neurol. 393, 353-373.

80. Sümbül, U., Song, S., McCulloch, K., Becker, M., Lin, B., Sanes, J.R., Masland, R.H., and Seung, H.S. (2014). A genetic and computational approach to structurally classify neuronal types. Nat. Commun. $5,3512$.

81. Taniguchi, H., He, M., Wu, P., Kim, S., Paik, R., Sugino, K., Kvitsiani, D., Fu, Y., Lu, J., Lin, Y., et al. (2011). A resource of Cre driver lines for genetic targeting of GABAergic neurons in cerebral cortex. Neuron 71, 995-1013.

82. Tavakoli, A., Schmaltz, A., Schwarz, D., Margrie, T.W., Schaefer, A.T., and Kollo, M. (2018). Quantitative association of anatomical and functional classes of olfactory bulb neurons. J. Neurosci. 38, 7204-7220.

83. Telley, L., Cadilhac, C., Cioni, J.-M., Saywell, V., Jahannault-Talignani, C., Huettl, R.E., SarrailhFaivre, C., Dayer, A., Huber, A.B., and Ango, F. (2016). Dual function of NRP1 in axon guidance and subcellular target recognition in cerebellum. Neuron 91, 1276-1291.

84. Thorndike, R.L. (1953). Who belongs in the family? Psychometrika 18, 267-276.

85. Tiklová, K., Björklund, Å.K., Lahti, L., Fiorenzano, A., Nolbrant, S., Gillberg, L., Volakakis, N., Yokota, C., Hilscher, M.M., Hauling, T., et al. (2019). Single-cell RNA sequencing reveals midbrain dopamine neuron diversity emerging during mouse brain development. Nat. Commun. 10, 581.

86. Trapnell, C., Cacchiarelli, D., Grimsby, J., Pokharel, P., Li, S., Morse, M., Lennon, N.J., Livak, K.J., Mikkelsen, T.S., and Rinn, J.L. (2014). The dynamics and regulators of cell fate decisions are revealed by pseudotemporal ordering of single cells. Nat. Biotechnol. 32, 381-386.

87. Wamsley, B., and Fishell, G. (2017). Genetic and activity-dependent mechanisms underlying interneuron diversity. Nat. Rev. Neurosci. 18, 299-309.

88. Wang, X., Tucciarone, J., Jiang, S., Yin, F., Wang, B.-S., Wang, D., Jia, Y., Jia, X., Li, Y., Yang, T., et al. (2019). Genetic Single Neuron Anatomy Reveals Fine Granularity of Cortical Axo-Axonic Cells. Cell Rep. 26, 3145-3159.e5.

89. Ward, J.H. (1963). Hierarchical Grouping to Optimize an Objective Function. J. Am. Stat. Assoc. 58, 236-244.

90. Wefers, A.K., Haberlandt, C., Tekin, N.B., Fedorov, D.A., Timmermann, A., van der Want, J.J.L., Chaudhry, F.A., Steinhäuser, C., Schilling, K., and Jabs, R. (2017). Synaptic input as a directional cue for migrating interneuron precursors. Development 144, 4125-4136.

91. Wefers, A.K., Haberlandt, C., Surchev, L., Steinhäuser, C., Jabs, R., and Schilling, K. (2018). Migration of interneuron precursors in the nascent cerebellar cortex. Cerebellum 17, 62-71.

92. Weisheit, G., Gliem, M., Endl, E., Pfeffer, P.L., Busslinger, M., and Schilling, K. (2006). Postnatal development of the murine cerebellar cortex: formation and early dispersal of basket, stellate and Golgi neurons. Eur. J. Neurosci. 24, 466-478.

93. Winnubst, J., Bas, E., Ferreira, T.A., Wu, Z., Economo, M.N., Edson, P., Arthur, B.J., Bruns, C., Rokicki, K., Schauder, D., et al. (2019). Reconstruction of 1,000 Projection Neurons Reveals New Cell Types and Organization of Long-Range Connectivity in the Mouse Brain. Cell 179, 268-281.e13.

94. Wolf, F.A., Angerer, P., and Theis, F.J. (2018). SCANPY: large-scale single-cell gene expression data analysis. Genome Biol. 19, 15.

95. Wu, H., Williams, J., and Nathans, J. (2012). Morphologic diversity of cutaneous sensory afferents revealed by genetically directed sparse labeling. Elife 1, e00181.

96. Zeisel, A., Hochgerner, H., Lönnerberg, P., Johnsson, A., Memic, F., van der Zwan, J., Häring, M., Braun, E., Borm, L.E., La Manno, G., et al. (2018). Molecular architecture of the mouse nervous system. Cell 174, 999-1014.e22.

97. Zeng, H., and Sanes, J.R. (2017). Neuronal cell-type classification: challenges, opportunities and the path forward. Nat. Rev. Neurosci. 18, 530-546.

98. Zhang, L., and Goldman, J.E. (1996). Generation of cerebellar interneurons from dividing progenitors in white matter. Neuron 16, 47-54. 
bioRxiv preprint doi: https://doi.org/10.1101/2020.02.29.971366; this version posted May 2, 2020. The copyright holder for this preprint (which was not certified by peer review) is the author/funder, who has granted bioRxiv a license to display the preprint in perpetuity. It is made available under aCC-BY-NC 4.0 International license.
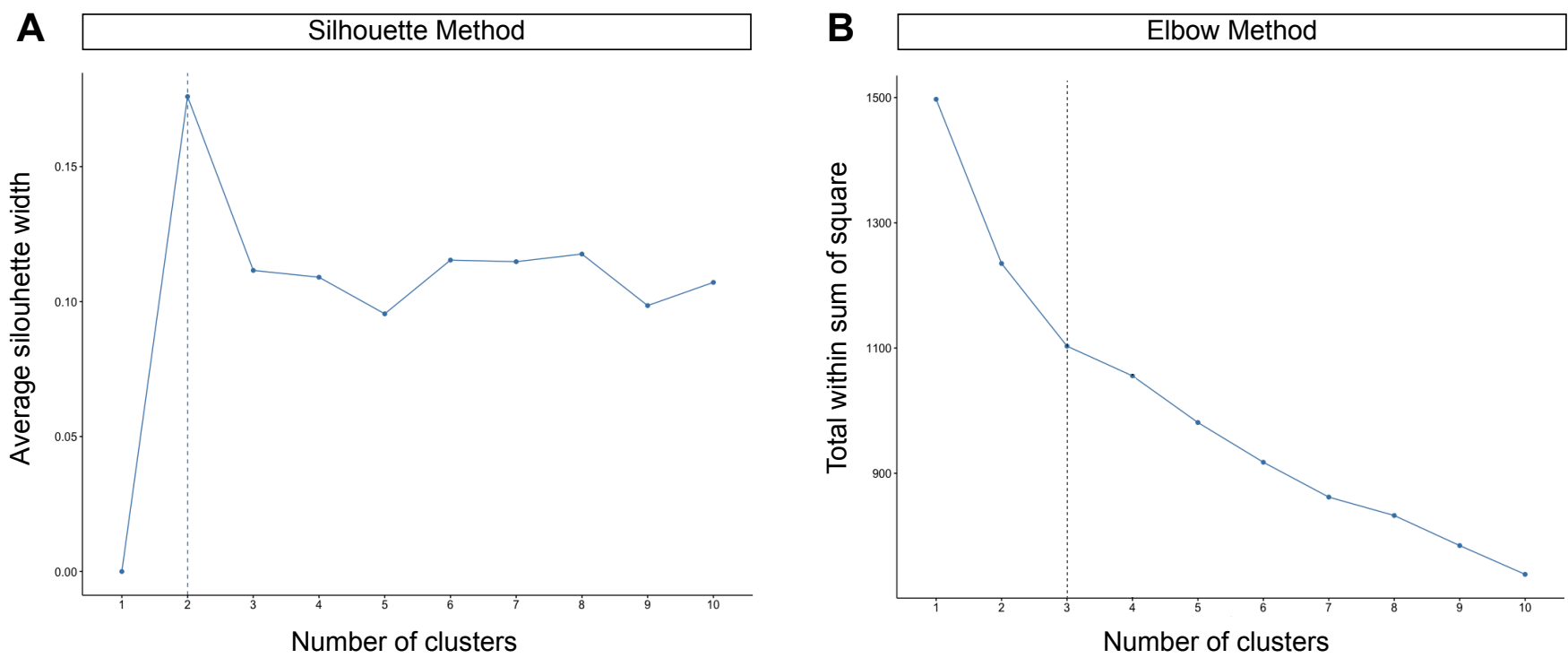

Supplementary Figure S1. Statistical confirmation for the optimal number of MLI clusters. (A) The silouhette method measures the quality of clustering by assessing how well each cell lies within their cluster. (B) The elbow method measures the k-means score and the total within sum of squares distance by assessing the distance of each cell to the cluster centroid. The optimal number of clusters is determined as 2 by the silouette method and 3 by the elbow method. 
bioRxiv preprint doi: https://doi.org/10.1101/2020.02.29.971366; this version posted May 2, 2020. The copyright holder for this preprint (which was not certified by peer review) is the author/funder, who has granted bioRxiv a license to display the preprint in perpetuity. It is made available under aCC-BY-NC 4.0 International license.
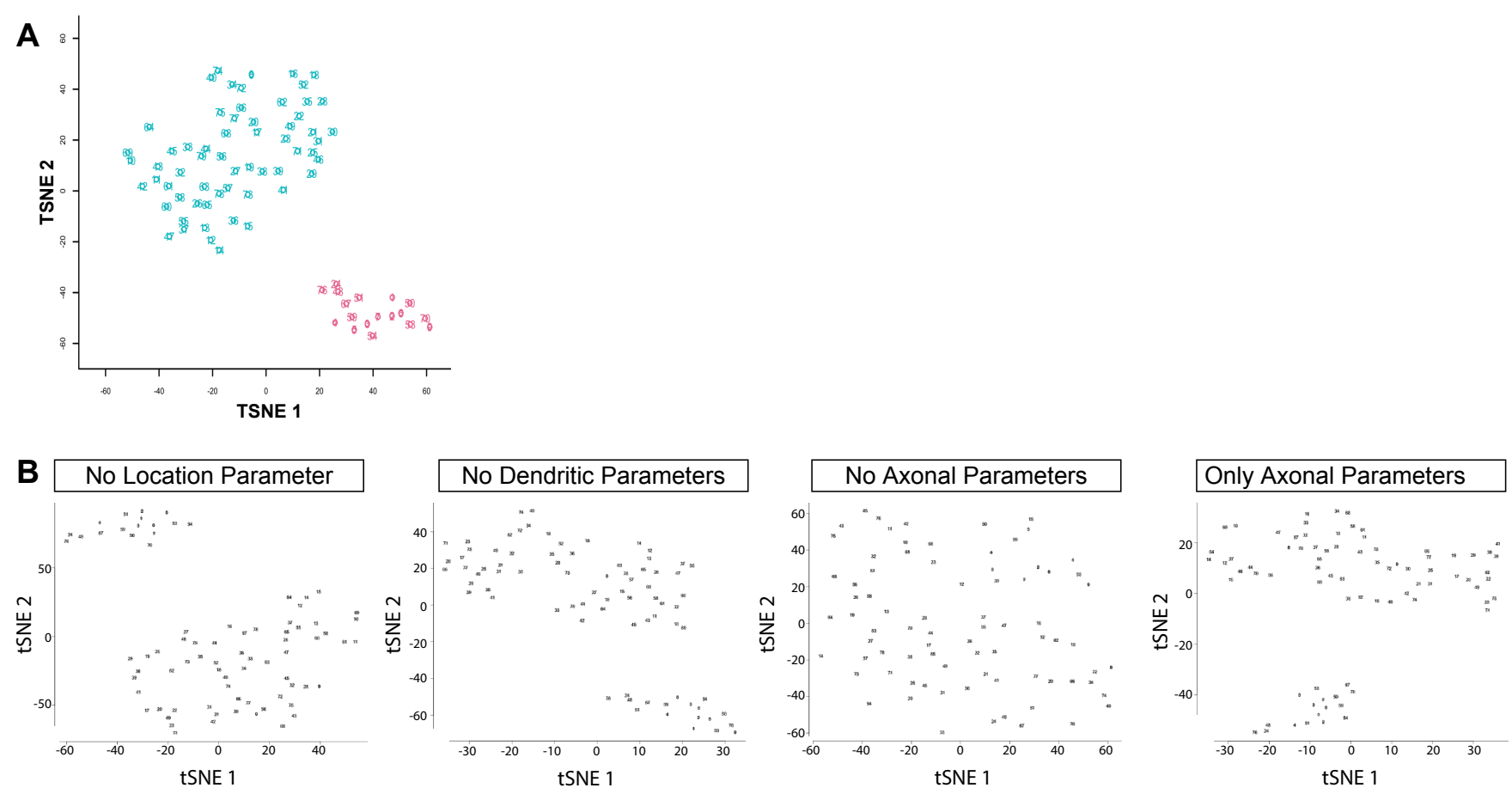

Supplementary Figure S2. Confirmation of UMAP results by t-SNE. (A) t-SNE of 79 mature MLIs. BCs are clustered in pink and SCs are colored in cyan. (B) Iterative feature elimination using t-SNE confirms that axonal information is necessary and sufficient for the BC/SC division. 
bioRxiv preprint doi: https://doi.org/10.1101/2020.02.29.971366; this version posted May 2, 2020. The copyright holder for this preprint (which was not certified by peer review) is the author/funder, who has granted bioRxiv a license to display the preprint in perpetuity. It is made available under aCC-BY-NC 4.0 International license.

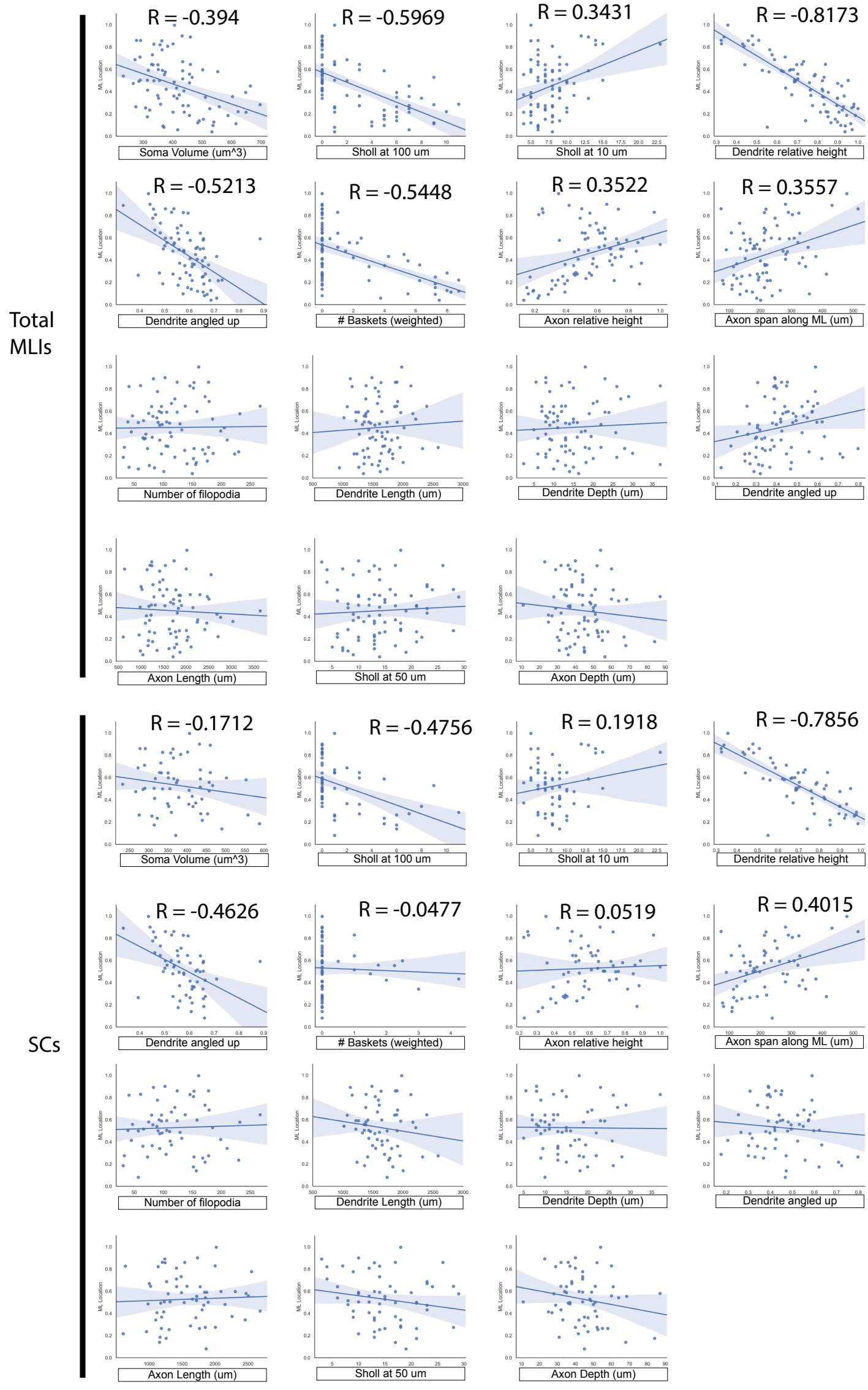

Supplementary Figure S3. SCs present significant within-class heterogeneity as confirmed through linear regression of morphological parameters. The top eight parameters show a postive or negative correlation to ML depth for the entire MLI population. 
bioRxiv preprint doi: https://doi.org/10.1101/2020.02.29.971366; this version posted May 2, 2020. The copyright holder for this preprint (which was not certified by peer review) is the author/funder, who has granted bioRxiv a license to display the preprint in perpetuity. It is made available under aCC-BY-NC 4.0 International license.
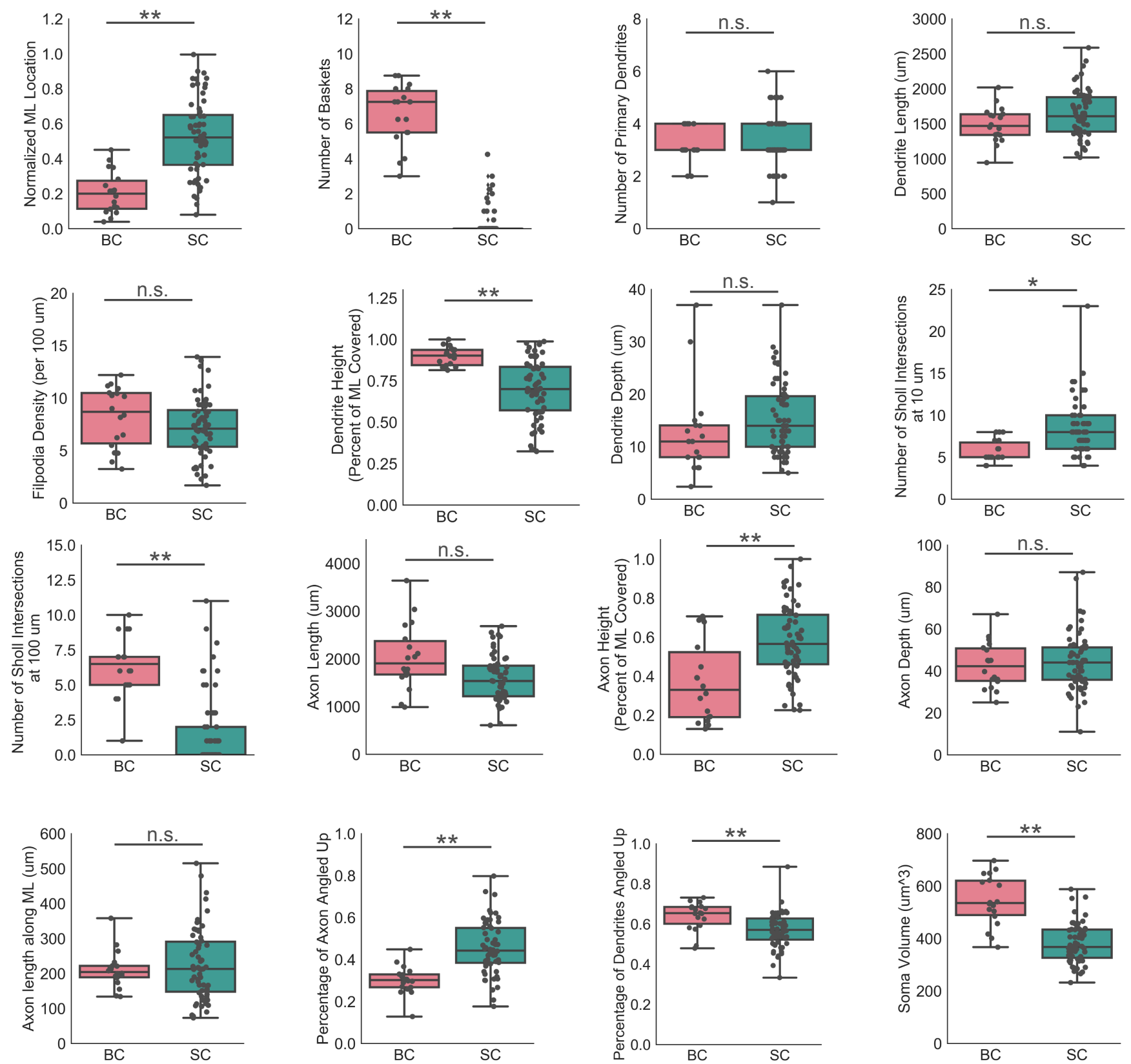

Supplementary Figure S4. Individual morphometric parameters for BCs and SCs, as per hierarchical clustering. All parameters overlap between BCs and SCs, and thus no individual parameter is sufficient for MLI subtype identification. The number of baskets is a good proxy. All parameters follow a largely normal distribution, with no presence of bimodality. Upper and lower whiskers of the box plots represent the maximum and minimum values of the dataset, respectively. The box is drawn connecting the two innermost quartiles. 
bioRxiv preprint doi: https://doi.org/10.1101/2020.02.29.971366; this version posted May 2, 2020. The copyright holder for this preprint (which was not certified by peer review) is the author/funder, who has granted bioRxiv a license to display the preprint in perpetuity. It is made available under aCC-BY-NC 4.0 International license.

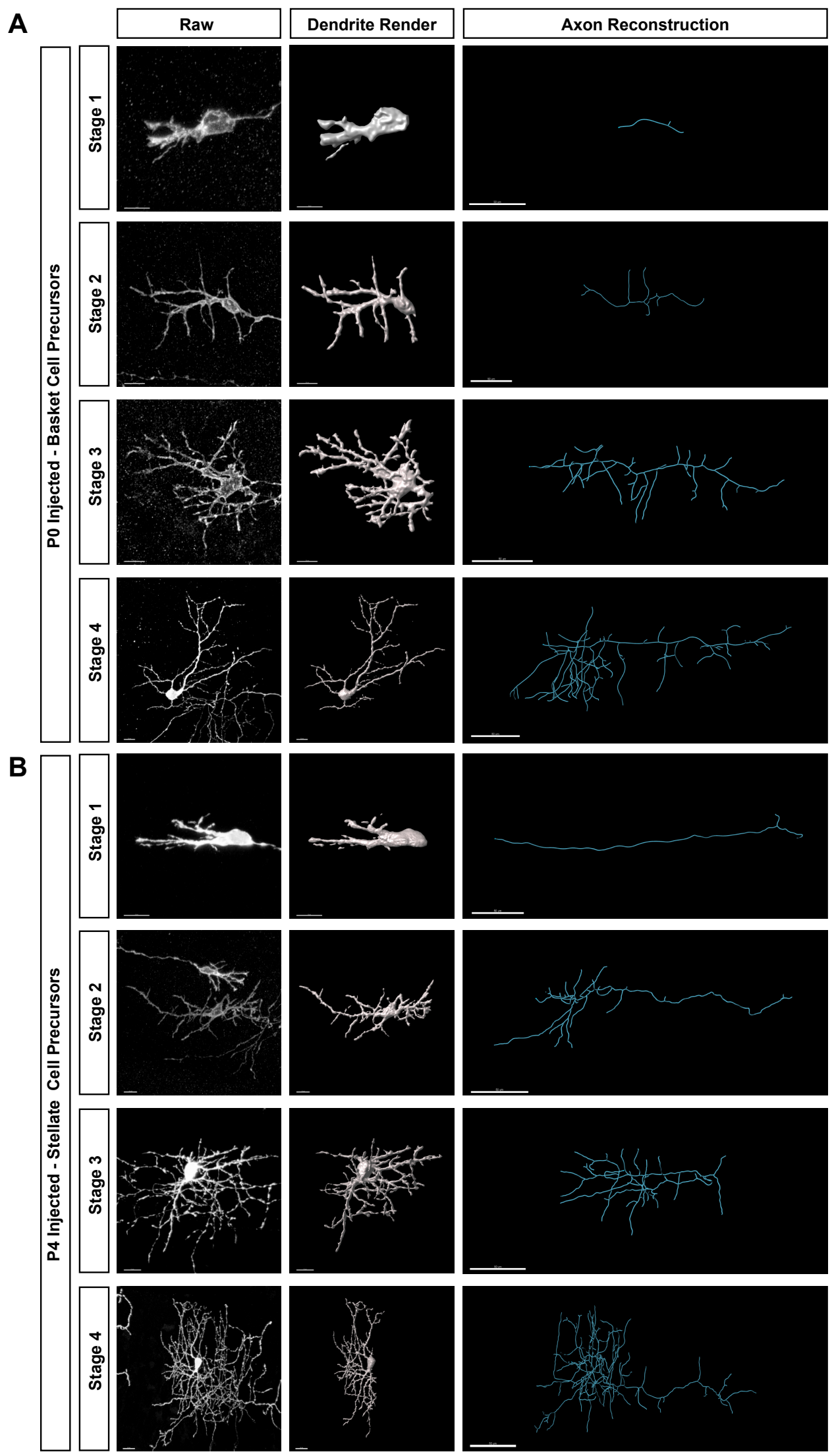

Supplementary Figure S5. Manual staging of MLI morphogenic maturation based on dendritic morphology for (A) early (P0) and (B) late-born (P4-7) MLIs. Stage 1 cells are migratory MLIs with clear migratory morphologies and leading processes. Stage 2 cells are maturing migratory MLIs with a developing dendritic arbor. Stage 3 cells have dendritic arbors of increasing complexity and rounded somas. Stage 4 cells are mature MLIs with type-specific dendritic and axonal arbors. 
bioRxiv preprint doi: https://doi.org/10.1101/2020.02.29.971366; this version posted May 2, 2020. The copyright holder for this preprint (which was not certified by peer review) is the author/funder, who has granted bioRxiv a license to display the preprint in perpetuity. It is made available under aCC-BY-NC 4.0 International license.

\section{A Curated Maturity Stage}

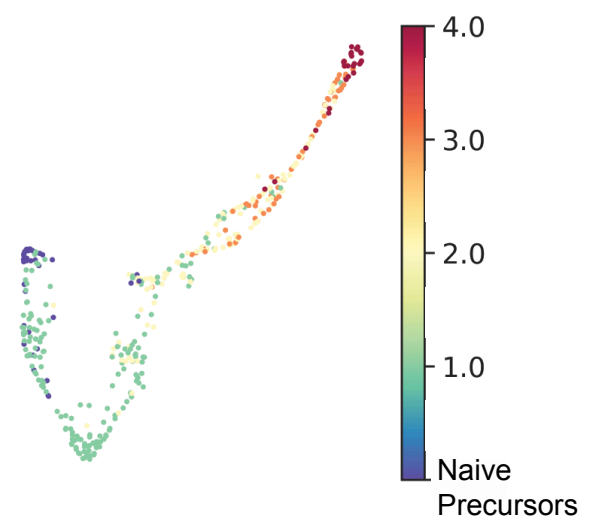

B

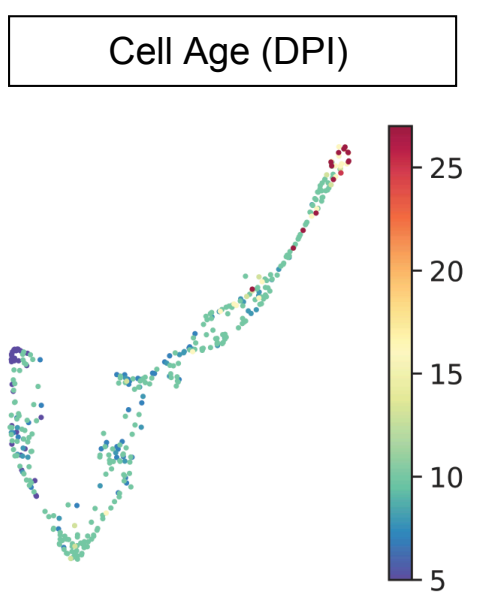

Supplementary Figure S6. (A) Curated maturity stage for single MLIs projected onto Palantir trajectory. Naive precursor cells correspond to figure $6 \mathrm{M}$, and are characterized as migratory MLIs with prominent leading process, but lacking visible trailing process. (B) Cell age projected onto Palantir trajectory, as inferred from days after tamoxifen injection (DPI). 
bioRxiv preprint doi: https://doi.org/10.1101/2020.02.29.971366; this version posted May 2, 2020. The copyright holder for this preprint (which was not certified by peer review) is the author/funder, who has granted bioRxiv a license to display the preprint in perpetuity. It is made available under aCC-BY-NC 4.0 International license.

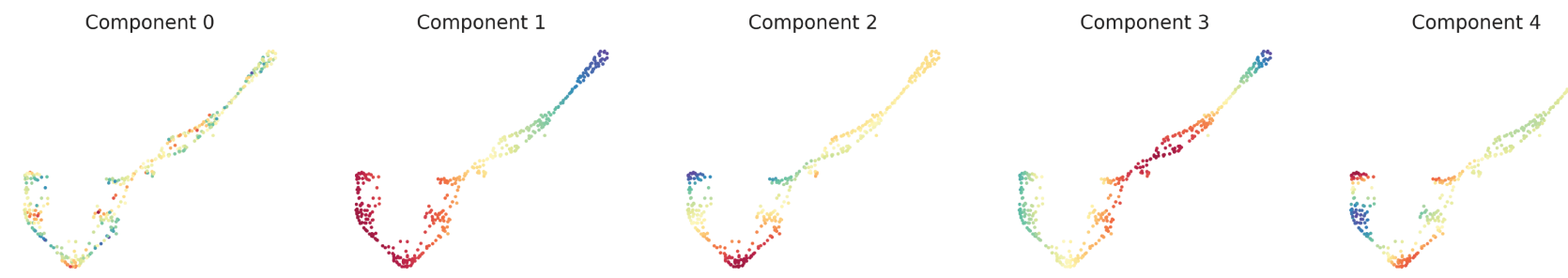

Supplementary Figure S7. Diffusion map components for early-born MLI Palantir trajectory. 
bioRxiv preprint doi: https://doi.org/10.1101/2020.02.29.971366; this version posted May 2, 2020. The copyright holder for this preprint (which was not certified by peer review) is the author/funder, who has granted bioRxiv a license to display the preprint in perpetuity. It is made available under aCC-BY-NC 4.0 International license.
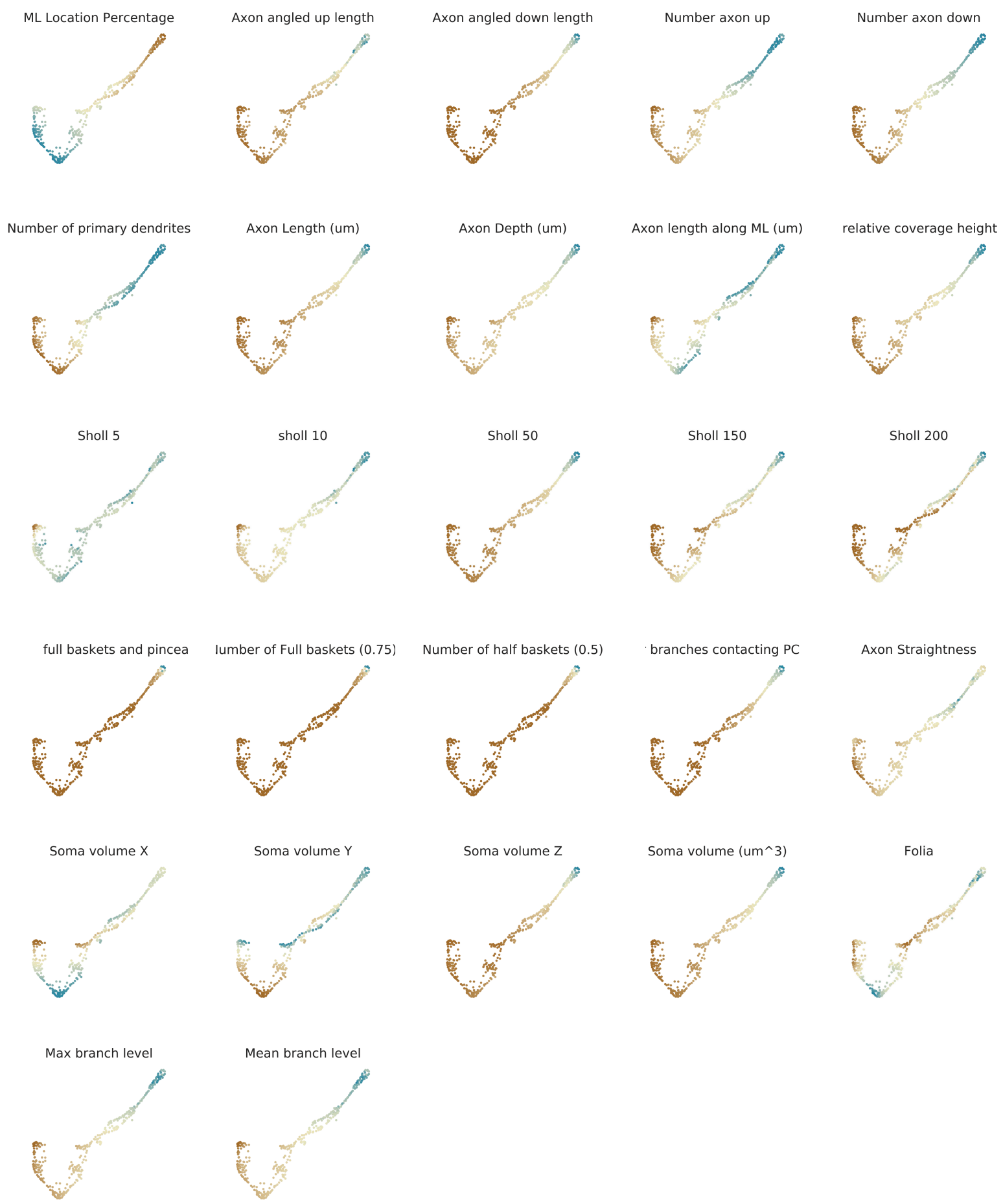

Supplementary Figure S8. Projection of single morphometric parameters onto Palantir pseudotime trajectory. Minimum parameter values are highlighted in brown and maximum parameter values are highlighted in blue. 
bioRxiv preprint doi: https://doi.org/10.1101/2020.02.29.971366; this version posted May 2, 2020. The copyright holder for this preprint (which was not certified by peer review) is the author/funder, who has granted bioRxiv a license to display the preprint in perpetuity. It is made available under aCC-BY-NC 4.0 International license.
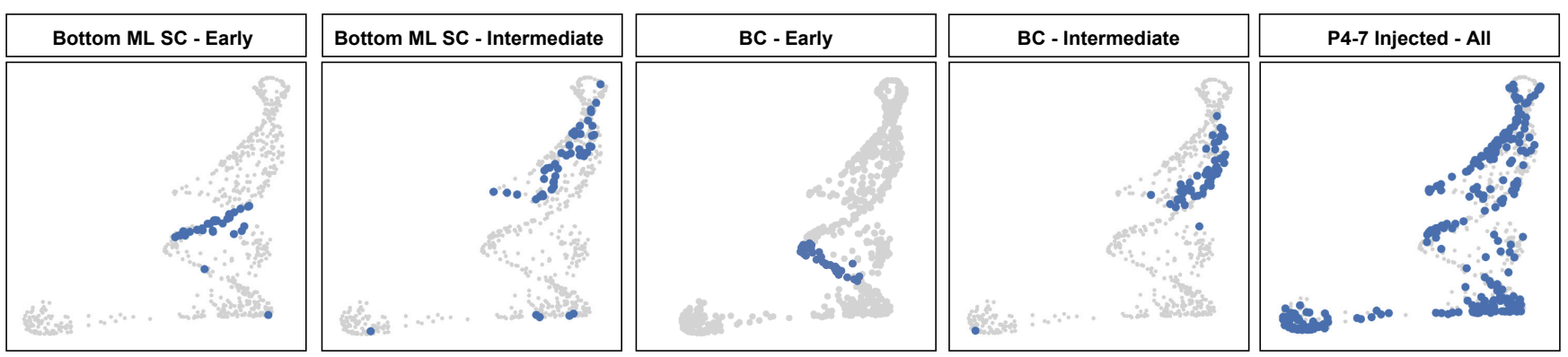

Supplementary Figure S9. MLI lineages form discontinuous trajectories by Palantir. Early and intermediate bottom ML SCs represent cluster 5 and 7 cells from Figure 6, respectively. Early and intermediate BCs represent cluster 3 and 6 cells from Figure 6 , respectively. Early BCs, bottom ML SCs, and middle/upper ML SCs are largely non-overlapping, suggesting subtype-specific clustering from the beginning of pseudotime. 
bioRxiv preprint doi: https://doi.org/10.1101/2020.02.29.971366; this version posted May 2, 2020. The copyright holder for this preprint (which was not certified by peer review) is the author/funder, who has granted bioRxiv a license to display the preprint in perpetuity. It is made available under aCC-BY-NC 4.0 International license.
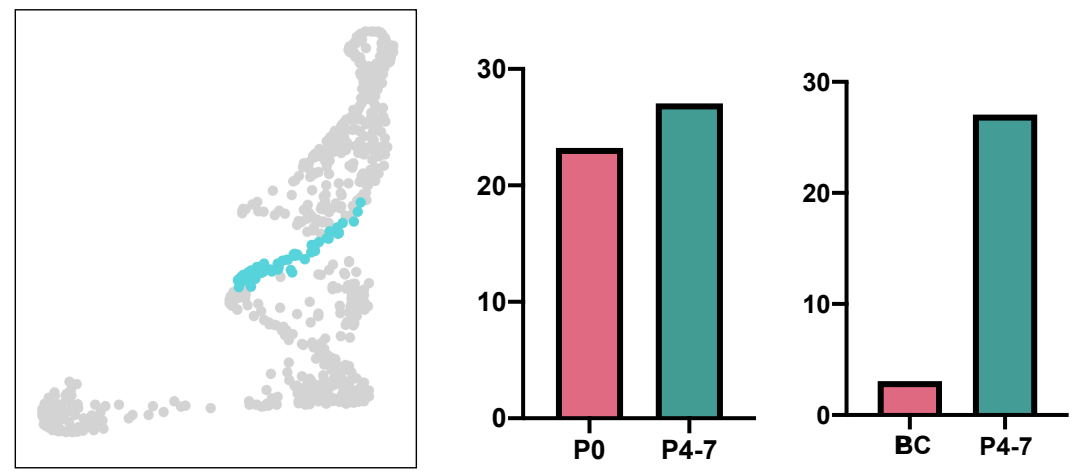

Supplementary Figure S10. Cluster 6 contains early-born SC1s. Cluster 6 contained similar numbers of early- and late-born MLIs. However, this is due to the the high enrichment of SC1s within this cluster, as seen after removing SC1s from the early born population (right). 